\title{
LA PROTECGIÓN SOCIAL EN SALUD DURANTE EL GOBIERNO DE CALDERÓN. AVANCES Y REZAGOS EN EL DISEÑOY LA IMPLEMENTACIÓN DEL SEGURO POPULAR (2006-2012)
}

LAURA FLAMAND

Carlos Moreno-Jaimes ${ }^{1}$

LA CAÍDA DEL PRI EN 2000 Y EL PRIMER GOBIERNo de Acción Nacional despertaron grandes expectativas de reformas en un amplio espectro de arenas de política (e.g. fiscal, industrial, política, de combate a la corrupción), sin embargo, la política social no parecía ocupar un lugar preeminente en la agenda del nuevo gobierno. Salvo el énfasis en transparentar el uso de los recursos destinados a los programas de combate a la pobreza para evitar que se emplearan con propósitos electorales.

Sin embargo, durante el sexenio de Fox se publicó la Ley General de Desarrollo Social (2003), donde se estableció que el gasto social tendría que aumentar cada año, por lo menos, proporcionalmente con la inflación, y se creó el Consejo Nacional de Evaluación de la Política Social, responsable de que estas políticas gubernamentales fuesen más transparentes y efectivas, con amplia participación ciudadana. Esta ley fundamenta el enfoque de derechos, la medición multidimensional de la pobreza y la evaluación permanente de los programas de desarrollo social que se consideran cambios centrales y positivos en la política social en México en este periodo. ${ }^{2}$

${ }^{1}$ Los autores agradecen el estupendo apoyo académico de Rafael Arriaga Carrasco.

${ }^{2}$ Ilán Bizberg y Laura Flamand, "Grandes tendencias y pequeños avances en 
Además de estos cambios, en el área de la política sanitaria, durante el gobierno de Fox se creó y se impulsó el Sistema de Protección Social en Salud con el Seguro Popular. La creación del Sistema, como detallamos más adelante, significó un reconocimiento muy claro de la desigualdad persistente entre los servicios para las personas con seguridad social y aquellos que atienden a la población no asegurada. La política que impulsaron Julio Frenk y su equipo, desde la Secretaría de Salud, se apoyó en los trabajos de investigación que se desarrollaron en la Fundación Mexicana de la Salud; dicha política enfatizaba la necesidad de proteger a la población más marginada del gasto de bolsillo en salud que, en muchas circunstancias, podía tornarse empobrecedor o, incluso, catastrófico.

Este artículo se ocupa de describir y analizar la evolución del Seguro Popular durante el gobierno de Felipe Calderón (20062012). El texto se organiza en cuatro secciones. En la primera, analizamos muy brevemente los énfasis de los gastos social y sanitario de este periodo. La segunda se ocupa de describir el surgimiento y las fases iniciales de Seguro Popular durante el gobierno de Fox. En la tercera sección destacamos las transformaciones y los alcances de la política durante la administración de Felipe Calderón, en particular: la meta de cobertura universal, la asignación financiera por persona y no por familia, así como la creación de las estrategias (Seguro Médico Nueva Generación y Embarazo Saludable). Para finalizar, en la cuarta sección, discutimos la relación entre el Seguro Popular y las propuestas de establecer un sistema universal de salud en México para enfatizar la necesidad de fortalecer los sistemas estatales de salud.

Nuestro análisis revela que el Seguro Popular surgió de un diagnóstico sólido y de una preocupación fundada sobre la necesidad de hacer efectiva la protección social en salud y de un gasto sanitario más equitativo. Sin embargo, sus efectos sobre el gasto de bolsillo y sobre los servicios de salud en general han resultado bastante limitados, ${ }^{3}$ en buena medida, porque el gasto público en salud

las políticas sociales (2000-2012)", en Ana Covarrubias (ed.), Doce años de gobierno del PAN, México, El Colegio de México, en dictamen.

${ }^{3}$ Como objetivo, en cuanto al gasto de bolsillo, proponemos que la propor- 
sigue siendo relativamente bajo y porque el carácter descentralizado de los servicios sanitarios que atienden a la población no asegurada no se ha considerado de manera cabal en la implementación. En consecuencia, los efectos positivos del Seguro Popular (en términos de un mayor uso de los servicios de salud, en especial, los preventivos, la cobertura efectiva y la calidad de la atención) presentan diferencias muy considerables entre las entidades.

\section{GASTO SOCIAL Y GASTO EN SALUD DURANTE EL GOBIERNO de Calderón}

En los últimos veinte años, en México, el gasto social se ha duplicado (6.7\% del PIB en 1991 a $11.3 \%$ en 2010, gráfica 1). Incluso con este incremento considerable, la proporción del piB destinada al gasto social en México es muy baja comparada con las de otros países de América Latina: en 2010, Argentina gastó 27.8\% del PIB, Brasil $26.4 \%$ y Chile $15.2 \%$. Si se analiza el gasto social per cápita, también se observa que México ha gastado menos que Argentina, Brasil o Chile durante el mismo periodo. ${ }^{4}$

Para comenzar con el análisis puntual sobre el gasto social en México, presentamos una gráfica que parte de la propuesta e información del Presupuesto de Egresos de la Federación. En la gráfica 2 se analiza el grupo funcional de desarrollo social del gasto programable que incluye el presupuesto destinado a funciones educativas, de salud, de seguridad social, de urbanización, vivienda y desarrollo regional, de asistencia social y de servicios de agua potable y alcantarillado. ${ }^{5}$

ción de este componente respecto al gasto total en salud en México sea comparable a la de otros países de niveles de desarrollo similar. Por el momento, este objetivo no se ha alcanzado aún; de acuerdo al Banco Mundial (2014), el gasto de bolsillo es de $44 \%$ en México, 32\% en Chile, 31\% en Brasil, 20\% en Argentina y $14.8 \%$ en Colombia.

${ }^{4}$ En 2010, el gasto per cápita en desarrollo social en México fue de 906 dólares de 2005, Chile 1276, Brasil 1437 y Argentina 1680 .

${ }^{5}$ El análisis comienza en 2003 para que los datos de la serie de tiempo sean comparables, pues a partir de este año se agregaron al grupo de desarrollo social 


\section{GrÁFICA 1}

Gasto público social como proporción del PIB América Latina y el Caribe (países seleccionados)

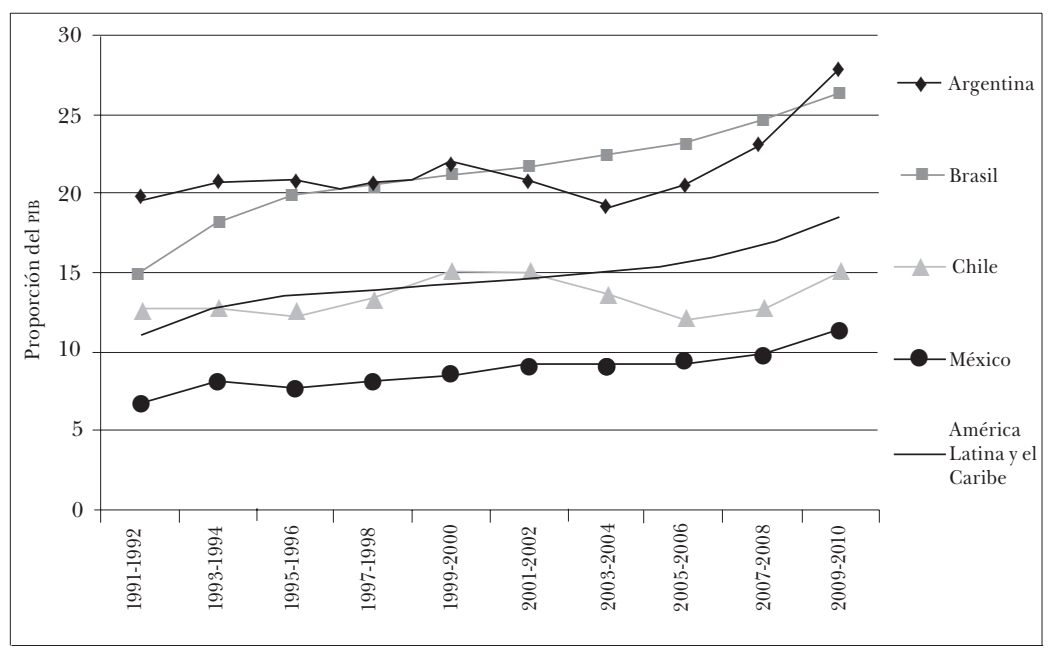

Fuente: Ilán Bizberg y Laura Flamand, "Grandes tendencias y pequeños avances en las políticas sociales (2000-2012)”, en Ana Covarrubias (ed.), Doce años de gobierno del PAN, México, El Colegio de México, en dictamen.con datos de la Comisión Económica para América Latina y el Caribe (CEPAL), Panorama social de América Latina, CEPAL, 2012, en http://www.eclac.org/publicaciones/xml/5/48455/ PanoramaSocial2012DocI-Rev.pdf

Notas: El dato de Brasil en 2010 corresponde a una estimación del gasto en las tres esferas de gobierno (federal, estadual y local) a partir de información oficial sobre gasto federal. Los datos para América Latina y el Caribe son promedios ponderados de 21 países por el tamaño de su producto interno bruto e incluyen estimaciones en años de países donde falta información.

La gráfica 2 confirma que el gasto social agregado se ha incrementado durante estos años. Sin embargo, obsérvese que mientras los recursos destinados a las funciones de salud y de educación se han mantenido estables, las proporciones de las otras funciones han crecido de manera sostenida en este periodo. La variación

las funciones de vivienda, agua potable y alcantarillado; además se eliminó la función laboral. 
más considerable corresponde a la función seguridad social, quizás a partir de la reforma a las pensiones del issste. ${ }^{6}$

GRÁFICA 2

Composición funcional del gasto en desarrollo social en México (2003-2010) (como porcentaje del PIB)

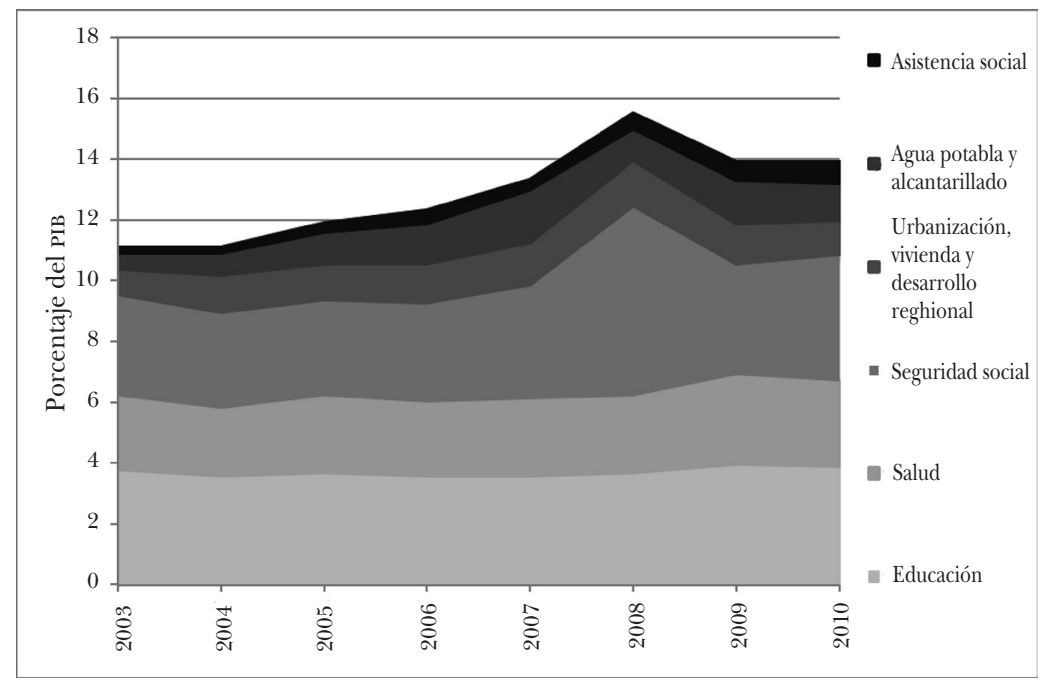

Fuente: Ilán Bizberg y Laura Flamand, "Grandes tendencias y pequeños avances en las políticas sociales (2000-2012)”, en Ana Covarrubias (ed.), Doce años de gobierno del Pan, México, El Colegio de México, en dictamen; con datos del Centro de Estudios de Finanzas Públicas (CEFP), Evolución del Gasto Social 2003-2010, México, CEFP, 2011, en http://www.cefp.gob.mx/intr/edocumentos/pdf/cefp/2009/cefp 0782009.pdf

Como detallaremos en las secciones siguientes, los énfasis y las acciones de la política social durante los dos sexenios de gobierno del PAN cambiaron de manera sustantiva. En particular, nótese, por

${ }^{6}$ Véase Miguel Ángel Yunes, La reforma del ISSSTE: un cambio necesario, México, FCE, 2009; también: Centro de Estudios Sociales y de Opinión Pública, Carpeta informativa. Iniciativa de reforma a la ley del ISSSTE, 2007, en http:/ / archivos.diputados. gob.mx/Centros_Estudio/Cesop/ISSSTE_2.pdf 
ejemplo, que los recursos del Seguro Popular han crecido de manera constante desde su creación en 2003. El presupuesto de Seguro Popular correspondía a $0.1 \%$ del gasto total en desarrollo social en 2003 y para 2010 llegó a 3\%, cada vez más cerca del presupuesto de Oportunidades $(3.5 \%)$.

\section{GRÁFICA 3}

Gasto público en salud como \% del PIв (1990-2010)

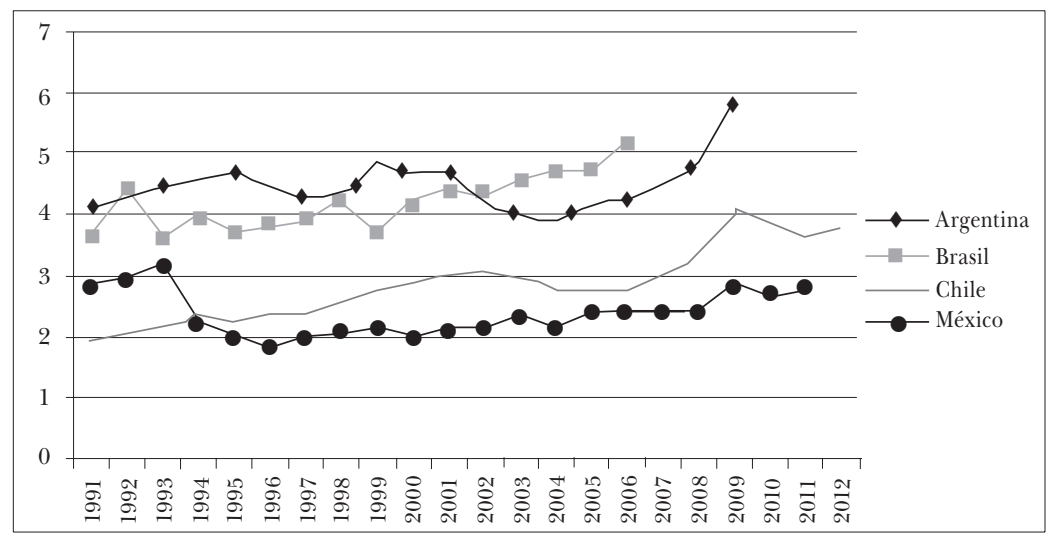

Fuente: Comisión Económica para América Latina y el Caribe (CEPAL), Gasto Social en América Latina y el Caribe, Portal de inversión social en la región, en http://dds.cepal.org/gasto/indicadores

Nota: De acuerdo a la disponibilidad de datos de la CEPAL y la naturaleza de los servicios de salud en cada país, los datos que se presentan en la gráfica corresponden al gobierno general de Argentina, al sector público no financiero de Brasil, al gobierno central de Chile y al gobierno central presupuestario de México. Conviene apuntar que, en el caso mexicano, las contribuciones de los gobiernos estatales al gasto total en salud son modestas, por tanto, incluir sólo al gobierno central presupuestario parece razonable, pues considera las transferencias federales condicionadas destinadas a la atención de la población no asegurada en las entidades.

Varios autores han advertido sobre los efectos acotados del Seguro Popular. ${ }^{7}$ Ilán Bizberg y Laura Flamand asocian estos efectos

${ }^{7}$ Véase Felicia Knaul, Eduardo González-Pier, Octavio Gómez Dantés et al., "The Quest for Universal Health Coverage: Achieving Social Protection for All in 
modestos a, entre otros factores, que el financiamiento público destinado a la salud no es suficiente para atender las necesidades sanitarias de los mexicanos sin seguridad social. ${ }^{8}$ Para el último año disponible, el gasto público en salud de México como proporción del PIB es menor al de Argentina y Chile (gráfica 3). De hecho, aunque el gasto público ha aumentado considerablemente, el privado (donde se incluye el gasto de bolsillo, con sus efectos perniciosos) representa todavía $78.8 \% .^{9}$

$\mathrm{Si}$ analizamos con más detalle otros indicadores del sistema de salud mexicano en paralelo a los de otros países en América Latina observamos una tendencia similar. Mientras el número de médicos por habitante en México mejoró considerablemente (de 828 personas por médico en 2000 a 641 en 2010), en cuanto a la infraestructura física hubo un retroceso (de 0.8 camas por 1000 habitantes en 2000, a 0.7 en 2010. En Brasil, en cambio hay 353 habitantes por médico y 3.4 camas por 1000 habitantes. ${ }^{10}$

\section{El surgimiento de Seguro Popular durante EL GOBIERNO DE FoX}

El gobierno federal mexicano desarrolló el Seguro Popular en 2003 para ofrecer protección financiera frente a los gastos de salud de la población excluida de la seguridad social, es decir más de cincuenta millones de mexicanos. ${ }^{11}$ La política, cuyo fundamento

Mexico", The Lancet, vol. 380, núm. 9849, 2012, en http://www.thelancet.com/ journals/lancet/issue/vol380no9849/PIIS0140-6736(12)X6041; también: Fundación Mexicana para la Salud (Funsalud), "Universalidad de los servicios de salud en México”, Salud Pública de México, vol. 55, 2013, pp. EE1-EE64.

${ }^{8}$ Bizberg y Flamand, art. cit.

${ }^{9}$ Consejo Nacional de Evaluación de la Política de Desarrollo Social (Coneval), Informe de Evaluación de la Política de Desarrollo Social en México 2012, México, Coneval, 2012, p. 191, en http://www.coneval.gob.mx/Informes/Evaluacion/ IEPDS2012/Pages-IEPDSMex2012-12nov-VFinal_lowres6.pdf

${ }^{10}$ Cepal, Statistical Yearbook for Latin America and the Caribbean, Santiago, Cepal, 2011, en http://www.eclac.cl/publicaciones/xml/7/45607/1.pdf

${ }^{11}$ El Seguro Popular inició en 2002 como un programa piloto que operó en cinco estados, aunque al entrar en vigor las reformas a la Ley General de 
institucional proviene de la reforma a la Ley General de Salud en $2003,{ }^{12}$ se propone resolver un problema central y persistente desde mediados de la década de 1990: casi $60 \%$ del gasto en salud en México provenía de fuentes privadas, pero fundamentalmente del bolsillo de las personas que requerían atender sus problemas de salud fuera del sistema público al momento de requerir atención. ${ }^{13}$

Es decir, el sistema de salud mexicano se financiaba, predominantemente, a través de un mecanismo ineficiente e inequitativo, pues el gasto de bolsillo rompe con el principio de justicia financiera, según el cual los individuos deben sufragar sus gastos sanitarios de acuerdo a sus ingresos y recibir servicios en función de sus necesidades de salud. El Seguro Popular, en consecuencia, tiene el objetivo fundamental de ofrecer protección financiera en salud a la población que carece de seguridad social con un esquema de aseguramiento público. De este modo, la política pública ataca el problema de empobrecimiento de las familias que enfrentan gastos en salud y fomenta una cultura de pago anticipado entre los beneficiarios. ${ }^{14}$ De manera sencilla, el Seguro Popular se ha propuesto disminuir el gasto de bolsillo como proporción del gasto total en salud.

Otro objetivo importante del Seguro Popular es lograr una distribución más equitativa de los recursos públicos destinados a atender

Salud en enero de 2004, la política sumó progresivamente a todas las entidades del país.

${ }^{12}$ Mauricio Ortiz (El Seguro Popular. Una crónica de la democracia mexicana, México, Secretaría de Salud-Funsalud-INSP-FCE, 2006) presenta una narrativa muy interesante sobre el proceso legislativo que permitió modificar la Ley General de Salud y crear el spss.

${ }^{13} \mathrm{El}$ gasto privado en salud, a grandes rasgos, se integra con los pagos a seguros y el gasto de bolsillo, es decir las erogaciones directas de los hogares para atender problemas de salud fuera del sistema público. Según la Organización Mundial de la Salud, los gastos de bolsillo o gastos directos en salud engloban todos los tipos de gastos sanitarios realizados en el momento en que el hogar se beneficia del servicio de salud. Organización Mundial de la Salud (oms), Informe sobre la salud en el mundo: la financiación de los sistemas de salud: el camino hacia la cobertura universal, Ginebra, oms en http://www.who.int/whr/2010/es/index.html

${ }^{14}$ Eduardo González Pier, Mariana Barraza, Cristina Gutiérrez y Armando Vargas, (coords.), Sistema de Protección Social en Salud: Elementos conceptuales, financieros y operativos, México, Secretaría de Salud, FCE, 2006, p. 27. 
la salud de la población asegurada y la no asegurada. Aunque esta última representa más de la mitad de los habitantes del país, el gasto público destinado a financiar los servicios de salud de este gran sector poblacional ha sido considerablemente menor comparado con los fondos destinados a la población con seguridad social (en 1994, el gasto público en salud como proporción del PIB fue cuatro veces mayor para los asegurados que para la población abierta). ${ }^{15}$ Como discutiremos más adelante, este desequilibrio ha comenzado a corregirse a partir de la instrumentación del Seguro Popular.

El sistema de protección social creado con la reforma a la Ley General de Salud en 2003 estableció un esquema financiero que rompe varias inercias del gasto. Primero, se distinguen dos tipos de servicios: los servicios de salud pública (por ejemplo la promoción de la salud, la vigilancia epidemiológica, así como la investigación y la información) y los servicios personales de atención a la salud, a los cuales se dirige el Seguro Popular. Segundo, se definió un conjunto básico de intervenciones de primer y segundo niveles, así como un paquete de alto costo de atención de tercer nivel, que los afiliados reciben en el momento de obtener atención médica, sin necesidad de desembolsar recursos propios que podrían representar un gasto catastrófico o empobrecedor para su economía familiar. ${ }^{16}$ Tercero, el Seguro Popular se propuso abandonar el modelo de financiamiento a la oferta, buscando financiar la demanda mediante el aseguramiento.

Conviene abundar sobre esta última característica, una de las innovaciones más significativas de Seguro Popular consistió en transformar la lógica del financiamiento público: en lugar de asignar los

${ }^{15}$ Calculado por los autores con información de Sistema Nacional de Información en Salud (sINAIs), Gasto público, 1990-2009 en: http://www.sinais.salud.gob. $\mathrm{mx} /$ recursosfinancieros/index.html

${ }^{16}$ El paquete de servicios de primer y segundo nivel de atención se denomina Catálogo Universal de Servicios de Salud (CAuses). Existe también un Fondo de Protección contra Gastos Catastróficos (FPGC) que otorga servicios médicos de alta especialidad a los afiliados del Seguro Popular que padecen enfermedades de alto costo. Felicia Knaul y Julio Frenk, "Health Insurance in Mexico: Achieving Universal Coverage through Structural Reform”, Health Affairs, 2005, vol. 24, núm. 6, pp. 1467-1476. 
recursos en función de la infraestructura médico-hospitalaria existente en cada entidad, tal y como había venido ocurriendo históricamente, los recursos se distribuyen en función del número de afiliados al Sistema con fórmulas que se establecen, de manera explícita, en la Ley General de Salud y en su Reglamento. ${ }^{17}$ Esta medida eliminó el sesgo favorable y sistemático hacia las regiones con mayor capacidad instalada, y, en cambio, asigna los recursos de acuerdo a las necesidades sanitarias de las entidades prestadoras de servicios, por lo menos respecto al número de personas que, en principio, deben atender.

Los servicios del Seguro Popular en cada entidad se financian con recursos de tres fuentes, cuyos montos se establecen de manera precisa en la Ley General de Salud. ${ }^{18}$ Las tres fuentes son la cuota social (CS), ${ }^{19}$ la aportación solidaria (As), que se divide en aportación solidaria estatal (ASE) y aportación solidaria federal (ASF),${ }^{20}$ y las cuotas individuales que los asegurados pagan de manera anticipada. Para finalizar, las cuotas individuales (es decir el

${ }^{17}$ La gran influencia de la capacidad instalada sobre la distribución regional del gasto en salud durante la década de los noventa se analiza en Carlos Moreno Jaimes, "La descentralización del gasto en salud en México: una revisión de sus criterios de asignación”, México, CIDE, Documento de Trabajo de la DAP, núm. 95, 2000.

18 Nelly Aguilera y Mariana Barraza-Llorenz, "FAssA: análisis sobre equidad y alternativas de asignación”, en Carlos Chiapa y César Velázquez (eds.), Estudios del Ramo 33, México, Coneval-Colmex, 2011.

${ }^{19}$ La cuota social corre a cargo del gobierno federal y equivale a $3.92 \%$ del salario mínimo general diario para el Distrito Federal por cada individuo afiliado. Antes de la última reforma a la Ley General de Salud en 2009, la cuota social se establecía, no por individuo, sino por familia afiliada (equivalía a $15 \%$ del salario mínimo por cada familia asegurada).

${ }^{20}$ La As se integra con las aportaciones concurrentes del gobierno federal y los gobiernos estatales. La ASE por persona afiliada debe corresponder, por lo menos, a la mitad de la cs, lo cual permite que los estados aporten más si lo desean. La ASF por persona debe representar, por lo menos, una y media veces el monto de la cs (Ley General de Salud, art. 77 bis 13, 2012). El monto de la ASF para cada entidad federativa se determina mediante una fórmula (definida en el Reglamento de la Ley General de Salud en Materia de Protección Social en Salud) que comprende los siguientes componentes de asignación: 1) número de familias beneficiarias, 2) necesidades de salud aproximadas mediante dos variables: probabilidad de muerte infantil y probabilidad de muerte en adultos, 3) aportaciones adicionales de las entidades y 4) desempeño de los servicios de salud. 
monto que cada persona paga en el momento de su afiliación al Seguro Popular) se establecen, de manera progresiva, en función del nivel de ingresos de los individuos. ${ }^{21}$ Se exenta de este pago a aquellas personas que pertenecen a los cuatro deciles más bajos de ingreso. En la práctica, sin embargo, el número de personas que paga una cuota acorde con sus ingresos mensuales para contar con la protección de Seguro Popular es muy bajo, aproximadamente $0.5 \%$ del total de afiliados en $2012 .{ }^{22}$

Es importante destacar que el Seguro Popular se diseñó como una política pública descentralizada que aprovecha la infraestructura existente y la capacidad de operación de las entidades federativas para extender su cobertura al mayor número posible de personas en todo el territorio nacional. El diseño descentralizado de Seguro Popular surge de los procesos de concurrencia y descentralización que avanzaron durante la década de 1990 a partir del Acuerdo Nacional para la Descentralización de los Servicios de Salud en 1996.23 En otros términos, el Seguro Popular se concibió para operar de acuerdo a un sistema de coordinación intergubernamental con la infraestructura, los medios y los procesos de cada uno de los sistemas estatales de salud (SESA).

En este esquema, la Secretaría de Salud del gobierno federal sería responsable de regular, coordinar, evaluar y supervisar las acciones de cada sistema estatal. Además, en cada entidad, se creó un régimen estatal de protección social en salud (REPSs) como instancia responsable de gestionar los servicios, administrar los recursos,

${ }^{21}$ Antes de la reforma de 2009 a la Ley General de Salud, esta cuota no era individual, sino familiar, aunque también su monto se establecía en función a la capacidad de pago de las familias aseguradas, Octavio Gómez Dantés, Sergio Sesma, Víctor Becerril et al., "Sistema de Salud en México", Salud Pública de México, 2011, vol. 53, núm. 2, pp. 220-233.

22 Auditoría Superior de la Federación, "Comisión Nacional de Protección Social en Salud. Seguro Popular. Auditoría de Desempeño”, en http://www.asf.gob. $\mathrm{mx} /$ Trans/Informes/IR2011i/Grupos/Desarrollo_Social/2011_0426_a.pdf

${ }^{23}$ Véase, por ejemplo, Guillermo Soberón et al., "Health Services Decentralization in Mexico: Formulation, Implementation and Results of Policy", Health Policy and Planning vol. 4, 1989, pp. 301-313; también: Laura Flamand, "Las perspectivas del nuevo federalismo: el sector salud. Las experiencias en Aguascalientes, Guanajuato y San Luis Potosí", cide-dTap, 55, México, 1997. 
supervisar a los prestadores de servicios y, muy especialmente, afiliar a la población elegible. A final de cuentas, aunque la propuesta original consideraba la posibilidad de comprar servicios a las instituciones de la seguridad social y a proveedores privados, la mayor parte de los servicios financiados con los recursos de Seguro Popular se ofrecen en los sistemas estatales de salud que operan los gobiernos estatales. Es decir, en la práctica, los proveedores principales de servicios para los afiliados de Seguro Popular son los SEsa. Muy probablemente, la idea de que el Seguro Popular contratara servicios a proveedores privados o a las instituciones de seguridad social se dejaron de lado frente al temor a una "privatización" de los servicios de salud. ${ }^{24}$

Es crucial reconocer que la decisión de descentralizar la operación de los servicios del Seguro Popular implicaba riesgos. El más importante, a nuestro juicio, fue que el ejercicio del derecho universal a la protección de la salud, consagrado en el artículo cuarto constitucional desde 1983, estuviese condicionado a las capacidades físicas, financieras, organizativas y de gestión de 32 sistemas estatales de salud diferentes y muy heterogéneos. En otras palabras, bajo el esquema descentralizado, la efectividad, la oportunidad y la calidad de los servicios de salud depende de la capacidad de cada entidad para cumplir con funciones tan esenciales como monitorear las condiciones de salud de la población, identificar riesgos sanitarios, informar sobre temas de salud, desarrollar políticas de salud pública y garantizar el acceso de las personas a servicios sanitarios individuales. ${ }^{25}$

En 2006, todas las entidades habían firmado acuerdos de coordinación con la Secretaría de Salud para participar en el Seguro

${ }^{24}$ Considérese, por ejemplo, la intervención de Ricardo Monreal (PRD), entonces gobernador de Zacatecas, en la firma del decreto que promulgó la nueva Ley General de Salud en 2003: "Debemos ser enfáticos y categóricos: no estamos de acuerdo con la privatización de la seguridad social y lejos está dicha reforma de pretender esa posibilidad" (Mauricio Ortiz, op. cit., p. 26).

${ }^{25}$ Los diez servicios de salud que se proponen como esenciales para cualquier sistema público pueden consultarse en National Public Health Performance Standards Program, "10 Essential Public Health Services", en http://www.cdc. gov/od/ocphp/nphpsp/essentialphservices.htm 
Popular, pues simplemente era imposible renunciar a los enormes recursos adicionales que el gobierno federal ofrecía para atender a la población no asegurada. Sin embargo, por ejemplo, las autoridades sanitarias del Distrito Federal se oponían en principio a la política con el argumento de que el spss contravenía los principios de gratuidad, universalidad y acceso irrestricto al derecho a la salud. ${ }^{26}$

Con todo, el Seguro Popular es la política pública más ambiciosa de los últimos años en materia de protección de la salud. Según los resultados de la Ensanut 2012, el spss se ha convertido en el principal asegurador público en México con $38.5 \%$ de la población ${ }^{27}$ y, además, su presupuesto ejercido en 2012 fue de 67500 millones de pesos, casi 17 veces más grande que en $2004 .^{28}$

\section{Transformaciones y algunos logros de Seguro Popular DURANTE EL GOBIERNO DE CALDERóN}

El gobierno del presidente Felipe Calderón continuó con el Seguro Popular retomando los elementos esenciales del esquema de protección social en salud que estableció la reforma a la Ley General de Salud de 2003, aunque con el propósito manifiesto de lograr la cobertura universal. ${ }^{29}$ Añadió, sin embargo, algunos aspectos nuevos que detallamos a continuación: Seguro Médico para una Nueva

${ }^{26}$ Mauricio Ortiz, op. cit., p. 94.

27 Juan Pablo Gutiérrez y Mauricio Hernández-Ávila, "Cobertura de protección en salud y perfil de la población sin protección en México, 2000-2012”, Salud Pública de México, vol. 55, supl. 2, 2013, pp. s83-s90.

${ }^{28}$ Datos de Comisión Nacional de Protección Social en Salud, "Informe de Resultados 2012", en http:/ / www.seguro-popular.salud.gob.mx/index.php?option= com_content\&view=article\&id=378\&Itemid=427

29 Según el Informe de Resultados de la Comisión Nacional de Protección Social en Salud correspondiente a 2006, las líneas generales de acción en materia de salud del gobierno de Calderón fueron lograr la cobertura universal en salud, garantizar el abasto y el suministro de medicamentos y otros insumos para la salud, llevar a cabo acciones continuas para mejorar la calidad en la prestación de los servicios, implementar programas de prevención de enfermedades y de promoción de la salud, construir y dar mantenimiento a la infraestructura en salud y establecer mecanismos de protección contra riesgos sanitarios. 
Generación, la Estrategia Embarazo Saludable y un énfasis renovado al Programa Nacional de Cirugía Extramuros.

Seguro Médico para una Nueva Generación $(S M N G) .{ }^{30}$ Esta iniciativa garantizó que, a partir del primero de diciembre de 2006, justo al inicio del sexenio de Calderón, todos los niños y niñas, a partir de su nacimiento, estuviesen cubiertos por un seguro médico amplio. Además de los beneficios y el financiamiento asociados al Seguro Popular, el SMNG incluye las intervenciones preventivas, curativas y de rehabilitación para todas las familias con niños nacidos a partir del primero de diciembre de 2006 afiliadas voluntariamente. ${ }^{31} \mathrm{El}$ SMNG se propuso garantizar a los niños el derecho a un seguro médico que cubriera todos los servicios médicos proporcionados en las unidades de salud, los medicamentos asociados, las consultas y los tratamientos, con la posibilidad de ser atendidos en sus localidades. En 2012, la Comisión Nacional de Protección Social en Salud reportaba una afiliación acumulada al sMNG de 5.3 millones de niños. ${ }^{32}$

Otras estrategias y programas asociados. Nuevos, también, dentro de la política de protección social en salud del gobierno de Calderón, fueron, por una parte, la Estrategia Embarazo Saludable (2008), que garantiza a toda mujer embarazada un seguro de atención médica para ella y su familia; ${ }^{33}$ por otra, el Programa Nacional Cirugía Extramuros, dirigido a la población de zonas de alta y muy alta marginación que no tienen acceso regular a servicios de

${ }^{30}$ A partir de 2013, en la administración de Peña Nieto, adoptó el nombre de Seguro Médico Siglo XXI.

${ }^{31}$ Las intervenciones de este programa (ahora Seguro Médico Siglo XXI) son acciones de segundo y tercer nivel de atención a la salud de los niños que no se incluyen en el CAUSES o en el FPGC. Estas acciones se cubren con el reembolso de cada intervención realizada a los beneficiarios del Seguro Médico Siglo XXI en la red de prestadores de servicios de salud en todo el país. Comisión Nacional de Protección Social en Salud (CNPss), "Informe de Resultados enero-junio 2013", en http://www.seguro-popular.salud.gob.mx/index.php?option=com_content\&v iew $=$ article $\& i d=378 \&$ Itemid $=427$, p. 71 .

${ }^{32}$ Ibid., p. 25.

${ }^{33}$ La afiliación a Embarazo Saludable ascendía en 2012 a 1.96 millones de mujeres, según datos de la Comisión Nacional de Protección Social en Salud, "Informe de Resultados enero-junio 2013...”, p. 26). 
cirugía. ${ }^{34}$ Finalmente, durante el gobierno de Calderón, el Seguro Popular comenzó a operar los servicios del componente de salud del Programa de Desarrollo Humano Oportunidades (heredado de las dos administraciones anteriores) llegando a cubrir a $15.3 \mathrm{mi}-$ llones de beneficiarios de ese programa en 2012. ${ }^{35}$

Cambios en el financiamiento. Un cambio importante ocurrió el 8 de diciembre de 2010, cuando el congreso federal aprobó una nueva reforma a la Ley General de Salud para modificar el mecanismo de financiamiento del Seguro Popular. Hasta esa fecha, el mecanismo de transferencia de recursos a las entidades se basaba en el número de familias aseguradas, lo cual, por ejemplo, generaba un incentivo para afiliar a personas mayores de 18 años que viven en el mismo hogar. De esta manera, se observó un aumento considerable en el número de familias pequeñas aseguradas al Seguro Popular, conque se decidió ajustar el esquema: en lugar de efectuar el pago por familia, a partir de la reforma, las transferencias responden al número de personas afiliadas. Con el nuevo mecanismo se intentó que la distribución de los recursos financieros fuese más equitativa y justa, pues el mecanismo anterior favorecía a las regiones más ricas, donde, en general, el tamaño de los hogares es menor. ${ }^{36}$

Además, se establecieron controles más estrictos sobre el gasto de las entidades. A partir de 2008, se estableció un límite máximo de $40 \%$ de los recursos federales para el pago de la nómina de salud, y otro de $30 \%$ para la adquisición de medicamentos, materiales y otros insumos. También se establecieron precios de referencia en la compra de medicamentos y se dispuso que ningún estado

${ }^{34}$ El Programa de Cirugía Extramuros se creó en 1991, sin embargo, durante el sexenio de Calderón comenzó a funcionar en el marco del spss, Secretaría de Salud, Cirugía extramuros: ¿Quiénes somos?, en http://www.salud.gob.mx/unidades/ $\mathrm{dgpfs} / \mathrm{micsitio} /$ cirextra/quienes/htmprese.htm

35 CNPss, op. cit., p. 29.

${ }^{36}$ Felicia Knaul, Eduardo González-Pier, Octavio Gómez-Dantés et al., "Hacia la cobertura universal en salud: protección social para todos en México", Salud Pública de México, vol. 55, núm. 2, 2013, pp. 207-235. 
podría financiar con recursos federales compras que rebasaran esos precios en más de $20 \% .{ }^{37}$

¿Cuáles han sido los principales logros del Seguro Popular, especialmente durante el gobierno de Calderón? Probablemente el resultado que más se ha aludido en las comunicaciones y la publicidad oficiales es el crecimiento de la afiliación y del gasto ejercido. Según datos del Quinto Informe de Gobierno, el presupuesto del Seguro Popular creció en consonancia con el aumento de la afiliación, tal y como se muestra en el cuadro 1, con la notable excepción de 2010, cuando la afiliación aumentó en casi 12.4 millones de personas respecto al año anterior, mientras que el presupuesto ejercido aumentó sólo en $18 \%$. La tasa anual de afiliación durante el periodo 2004-2012 fue, en promedio, de 5.4 millones de nuevos asegurados por año.

En 2012, el gobierno de Calderón declaró que se había alcanzado la cobertura universal, pues el Seguro Popular llegó a su meta de cobertura de 52.6 millones de personas. Sin embargo, el objetivo de corresponsabilizar a los afiliados a través de sus contribuciones directas al Seguro Popular no se ha alcanzado, pues el promedio de cuotas captadas por beneficiario disminuyó con el paso del tiempo: de casi 12 pesos en 2004 a 80 centavos en 2012 (véase el cuadro 1). Otro resultado con difusión muy amplia en los medios oficiales fue el crecimiento significativo del número de intervenciones de los niveles primero y segundo que se incluyen en el Catálogo Universal de Servicios de Salud (CAuses). Las intervenciones cubiertas por el Seguro Popular a través del Catálogo Universal pasaron de 90 en 2004 a 249 en 2006 y 284 en 2012.

Los datos oficiales de cobertura del Seguro Popular no suelen coincidir con los datos publicados por otras fuentes. En 2010, la Comisión Nacional de Protección Social en Salud reportaba 43.5 millones de afiliados, mientras que el Censo Nacional de Población registró 26.2 millones de personas que declararon estar afiliados al Seguro Popular. También, los cálculos de Coneval, a partir de la Encuesta Nacional de Ingreso y Gasto de los Hogares, revelaron que 35.8 millones de personas reportaron no tener derecho o 
adscripción a recibir servicios médicos de institución alguna, incluyendo el Seguro Popular. ${ }^{38}$ En 2012 las diferencias persistían, la Comisión Nacional reportó 52.7 millones de afiliados, mientras que, a partir de la Encuesta Nacional de Salud y Nutrición 2012 (Ensanut), se calcula que 44.3 millones de personas se encuentran adscritas al Seguro Popular. ${ }^{39}$

Estas diferencias en los datos responden a varias razones. Primero, es posible que, dado que la afiliación a Seguro Popular es familiar, algunos miembros del hogar no estén al tanto de este beneficio y, en consecuencia, no lo reporten a los encuestadores. Segundo, parte de la discrepancia puede explicarse por las personas que dicen reciben servicios de los sistemas estatales de salud, del Seguro Médico Nueva Generación o de la Estrategia Embarazo Saludable. Tercero, como el padrón de afiliados al Seguro Popular se construye de manera descentralizada a partir de los padrones estatales y las entidades tienen incentivos para afiliar al mayor número de personas (pues reciben recursos financieros por cada una), es probable que el padrón registre como afiliadas a personas que cuentan con servicios de salud de la seguridad social.

En cualquier caso, estas discrepancias significan un reto para el Seguro Popular, ya sea en términos de promoción entre sus afiliados o de mecanismos para depurar su padrón. De hecho, la evidencia disponible favorece la tercera hipótesis: un análisis a partir del Sistema Integral de Información de Padrones de Programas Gubernamentales (Secretaría de la Función Pública) revela que, en el primer semestre de 2013, había 6145428 personas

38 Coneval, Anexo Estadístico. Medición de la pobreza, Estados Unidos Mexicanos, 2010, en http://web.coneval.gob.mx/Paginas/principal.aspx; Instituto Nacional de Estadística y Geografía, Principales resultados del Censo de Población y Vivienda 2010, en http://www.inegi.org.mx/default.aspx; cNPss, Informe de resultados 2009, en http://www.seguro-popular.salud.gob.mx/index.php?option=com_content\&v iew=article \&id=378\&Itemid=427; CNPSs, Informe de transferencias a las entidades federativas 2010, en www.seguro-popular.salud.gob.mx; sinaIs, Boletín de Información Estadística, en http://www.sinais.salud.gob.mx/publicaciones/bie.html; sinaIs, Poblaciones 1990-2012 (total y condición de aseguramiento), en http://www.sinais.salud.gob.mx/demograficos/index.html

${ }^{39}$ Alejandra Macías, "Estado actual de la Seguridad Social en México, 2013", México, Centro de Investigación Económica y Presupuestaria, en ciep.mx/H0sWg 
que aparecían tanto en el padrón de Seguro Popular como en el del Imss. ${ }^{40}$

\section{CUADro 1}

Afiliación y gasto ejercido por Seguro Popular, 2004-2012.

Millones de pesos

\begin{tabular}{|c|c|c|c|}
\hline Año & Población afiliada & $\begin{array}{c}\text { Recursos ejercidos (millones } \\
\text { de pesos corrientes) }\end{array}$ & $\begin{array}{c}\text { Promedio de cuotas } \\
\text { captadas por beneficiario } \\
\text { afiliado (pesos) }\end{array}$ \\
\hline 2004 & 5318289 & 3462.80 & 11.77 \\
\hline 2005 & 11404861 & 6382.50 & 12.37 \\
\hline 2006 & 15672374 & 12170.40 & 8.98 \\
\hline 2007 & 21834619 & 18864.30 & 5.32 \\
\hline 2008 & 27176914 & 24915.60 & 8.41 \\
\hline 2009 & 31132949 & 31275.30 & 5.84 \\
\hline 2010 & 43518719 & 37029.50 & 4.88 \\
\hline 2011 & 51823314 & 45165.80 & 3.56 \\
\hline 2012 & 52738355 & 22042.80 & 0.80 \\
\hline
\end{tabular}

Nota: Las cifras del 2012 son al 30 de junio.

Fuente: Presidencia de la República, Sexto Informe de Gobierno, 2012. Anexo Estadístico, en http://calderon.presidencia.gob.mx/

Sin embargo, más allá del innegable crecimiento de la afiliación y el gasto, es preciso preguntarse si el Seguro Popular consiguió mejorar otros indicadores que atienden a su objetivo explícito: ofrecer protección social, en especial financiera, en salud. Las dimensiones que se han evaluado desde el inicio de la política en 2004 hasta el último año del sexenio de Calderón son la eficiencia y la equidad en la distribución de los recursos, la incidencia sobre

${ }^{40}$ Sistema Integral de Información de Padrones de Programas Gubernamentales (sıIPP-G), Unidad de Evaluación de la Gestión y el Desempeño Gubernamental, Secretaría de la Función Pública, Resumen de hallazgos y resultados primer semestre 2013, en http://www.funcionpublica.gob.mx/web/doctos/ua/ssfp/uegdg/siippg/resumen_siippg_1er_semestre_2013_ace.pdf 
el gasto de bolsillo de sus afiliados, la cobertura efectiva y la calidad de los servicios financiados con sus recursos.

En primer lugar, como ya hemos mostrado, la nueva política de protección social en salud consiguió elevar el monto del gasto en materia sanitaria. Como se muestra en el cuadro 2, el gasto total en salud como porcentaje del рів pasó de $5.1 \%$ en 2000 a $6.3 \%$ en 2010. Sin embargo, pese a dicho incremento, como ya hemos mencionado, el gasto público en salud como proporción del PIв en México (2.8\% del PIв) sigue estando muy por debajo de otros países de América Latina, por ejemplo, Argentina (5.9\%) y Chile $(4.1 \%){ }^{41}$

En segundo lugar, el Seguro Popular ha contribuido a disminuir el gasto de bolsillo en salud de las personas como porcentaje del gasto total sanitario: pasó de $52.9 \%$ en 2004 a $49.0 \%$ en $2011 .{ }^{42} \mathrm{Va}-$ rios estudios han mostrado el efecto protector del Seguro en cuanto a los gastos de bolsillo y el catastrófico. ${ }^{43}$ A partir de la Ensanut 2012, Ávila y coautores confirman los resultados, ${ }^{44}$ pero con matices importantes: los efectos protectores del Seguro Popular dependen del nivel socioeconómico (e. g. el efecto sobre el gasto excesivo en salud es menor entre los hogares más vulnerables) y de la zona geográfica (e.g. no tiene efecto sobre la probabilidad de gasto en salud

${ }^{41}$ Véase la gráfica 3, los datos corresponden a 2009.

42 Organization for Economic Co-operation and Development (OECD), Health Data 2013, en http://www.oecd.org/els/health-systems/oecdhealthdata2013-frequentlyrequesteddata.htm

${ }^{43}$ Omar Galárraga, Sandra Sosa-Rubi, Aarón Salinas-Rodríguez y Sergio SesmaVazquez, "Health Insurance for the Poor: Impact on Catastrophic and Out-of-Pocket Health Expenditures in Mexico", European Journal of Health Economics, vol. 11, núm. 5, 2010, pp. 437-447; Sandra Sosa-Rubi, Aarón Salinas-Rodriguez y Omar Galárraga, "Impacto del Seguro Popular en el gasto catastrófico y de bolsillo en el México rural y urbano, 2005-2008”, Salud Pública de México, vol. 53, 2011, pp. 425-435.

${ }^{44}$ En todo el país, $52 \%$ de los hogares tienen algún gasto en salud y $4.2 \%$ incurrieron en gasto excesivo en salud (mayor o igual a $30 \%$ del gasto total del hogar). El resultado más llamativo de este estudio es que la media del gasto en salud anual fue significativamente menor en hogares afiliados al Seguro Popular (200.2 pesos) que en hogares no afiliados (2400 pesos). Leticia Ávila-Burgos, Edson Serván-Mori, Verónica Wirtz, Sandra Sosa-Rubi y Aaron Salinas-Rodríguez, "Efectos del Seguro Popular sobre el gasto en salud en hogares mexicanos a diez años de su implementación”, Salud Pública de México, vol. 55, suplemento 2, 2013, pp. s91-s99. 
en zonas rurales, pero afecta el gasto excesivo en salud y el monto gastado). Grogger y coautores encuentran que el Seguro Popular ha reducido los gastos catastróficos en salud tanto en las zonas urbanas como en las rurales. ${ }^{45}$ En las segundas, sin embargo, los efectos varían dependiendo del tipo de unidad médica: los afiliados que sólo pueden acudir a instalaciones con personal médico insuficiente o poco calificado han experimentado reducciones muy pequeñas, casi insignificantes. En resumen, si bien los logros anteriores son dignos de reconocimiento, el gasto de bolsillo como proporción del total del gasto en salud en México (44.1\% en 2012) sigue siendo muy superior en comparación con los tres países de América Latina aludidos antes. ${ }^{46}$

En tercer lugar, la política de protección social en salud contribuyó a lograr un equilibrio más justo en la aplicación de los recursos públicos entre la población asegurada (i.e. los trabajadores asalariados y sus familias) y la no asegurada (i.e. la población objetivo del Seguro Popular). Como también se indica en el cuadro 2, a principios de la década pasada, por cada peso gastado en la población no asegurada, la población protegida por la seguridad social recibía más del doble, sin embargo, en 2010, el gasto destinado a la población asegurada fue sólo $20 \%$ superior al ejercido entre los no asegurados.

El gasto en salud financiado con recursos federales también ha mejorado su distribución territorial, pues la brecha que separa a la entidad más favorecida de la que recibe menos recursos federales se ha reducido a la mitad (la razón era 6.1 a 1 en 2000, 3 a 1 en 2010). Otro resultado positivo es que la contribución de los estados al financiamiento de la salud prácticamente se triplicó, en términos reales, entre 1999 y 2009, según cifras del Sistema Nacional

${ }^{45}$ Jeoffrey Grogger, Tamara Arnold, Ana Sofía León y Alejandro Ome, "Heterogeneity in the Effect of Public Health Insurance on Catastrophic Out-of-Pocket Health Expenditures: The Case of Mexico", Health Policy and Planning, junio de 2014, pp. 1-7.

${ }^{46} \mathrm{El}$ gasto de bolsillo como porcentaje del gasto total en salud en Argentina, Brasil y Chile asciende a 20.1, 31.0 y $32.1 \%$, respectivamente (Banco Mundial, 2014, World Development Indicators, disponible en http:/ / data.worldbank.org/datacatalog/world-development-indicators 
de Información en Salud (sinais). ${ }^{47}$ Las crecientes aportaciones estatales son consecuencia, muy probablemente, de los incentivos que se establecieron en el mecanismo de financiamiento del Sistema de Protección Social en Salud. ${ }^{48}$ Finalmente, conviene enfatizar que el porcentaje del presupuesto público federal destinado a la inversión en salud se ha elevado de $3.3 \%$ en 2000 a $4.4 \%$ en 2010 .

En cuanto a cobertura efectiva, los trabajos de Sosa y colaboradores ${ }^{49}$ muestran resultados promisorios. El Seguro Popular favorece el uso de los servicios de salud y el estado de salud en personas con diabetes; además, incrementa el uso de servicios hospitalarios públicos para atenciones obstétricas. Conviene resaltar, también, que los servicios estatales de salud, principales proveedores para los afiliados al Seguro Popular, concentraron la mayor proporción de las hospitalizaciones con $38.3 \%$ en $2012 .{ }^{50}$

Para concluir este apartado sobre resultados, es preciso señalar que la evidencia sobre la calidad de los servicios de salud que se financian con el Seguro Popular (y cuya provisión, como ya mencionamos en la sección anterior, se encuentra en manos de los 32 sistemas estatales de salud) no es concluyente. Por una parte, la evaluación de Emmanuela Gakidou et al. ${ }^{51}$ demuestra que la percepción de calidad de la atención no varía entre los afiliados al Seguro Popular y los no asegurados. Por otra, Felicia Knaul y Julio

47 SINAIS, Indicadores de recursos financieros, 1990-2009, en http://www.sinais. salud.gob.mx/recursosfinancieros/index.html

${ }^{48}$ Los gobiernos estatales sólo reciben el equivalente a la aportación solidaria federal una vez que han depositado la aportación solidaria estatal para financiar el sPss en su territorio.

${ }^{49}$ Sandra Sosa-Rubí, Omar Galárraga, Jeffrey Harris, "Heterogeneous Impact of the «Seguro Popular» Program on the Utilization of Obstetrical Services in Mexico, 2001-2006: A Multinomial Probit Model with a Discrete Endogenous Variable", Journal of Health Economics, vol. 28, núm. 1, 2009, pp. 20-34.

${ }^{50}$ Ávila-Burgos et al., op. cit.

${ }^{51}$ Emmanuela Gakidou, Rafael Lozano, Eduardo González-Pier, Jesse AbbottKlafter, Jeremy Barofsky, Chloe Bryson-Cahn, Deniss Feehan, Diana Lee, Héctor Hernández-Llamas y Christopher Murray, "Evaluación del impacto de la reforma mexicana de salud 2001-2006: un informe inicial”, Salud Pública de México, vol. 49, sup. 1, 2007, pp. 88-109. 
Frenk ${ }^{52}$ sostienen lo contrario, a partir de la alta tasa de reafiliación al programa entre 2003 y 2004, pues la consideran un indicador aproximado de satisfacción con los servicios. Grogger y coautores sostienen que todavía no hay evidencia de que la intervención haya mejorado la salud o haya incrementado la utilización de servicios médicos. ${ }^{53}$ En 2012, además, la Encuesta Nacional de Salud y Nutrición (Ensanut) reveló incrementos muy modestos en la proporción de personas que perciben que los servicios de salud de las unidades de la Secretaría de Salud son de buena calidad (78\% en 2006, 83\% en 2012), y en la proporción de usuarios que reportan mejoras en su salud ( $74 \%$ en 2006 y $78 \%$ en 2012 ).

En cuanto a la calidad, conviene resaltar, nuevamente, la heterogeneidad entre las entidades. La Ensanut 2006 reporta que, en todo el país, una proporción elevada de personas consideraban que la atención recibida era buena o muy buena $(81.1 \%)$, aunque con variaciones importantes: desde Quintana Roo con $77 \%$ hasta Nayarit con $86.5 \%$. Considérese, sin embargo, que las evaluaciones de los usuarios sobre la calidad de la atención de los sistemas de salud en México suelen ser muy favorables, tanto aquéllos para población con seguridad social ${ }^{54}$ como los servicios para población abierta, incluyendo, desde luego, los del Seguro Popular. ${ }^{55}$

${ }^{52}$ Knaul y Frenk, "Health Insurance in Mexico...".

${ }^{53}$ Jeoffrey Grogger, Tamara Arnold, Ana Sofía León y Alejandro Ome, "Efectos del Seguro Popular sobre el gasto en salud, utilización de servicios médicos y nivel de salud, y resultados de largo plazo del experimento del Seguro Popular en México y evidencia de la Encuesta Nacional de Ingresos y Gastos de los Hogares (ENIGH)", México, CIDE, 2011.

${ }^{54}$ Instituto Mexicano del Seguro Social (IMss), Encuesta Nacional de Satisfacción de Derechohabientes Usuarios de Servicios Médicos del IMSS, 2011 en http://www.imss. gob.mx/servicios/encuesta/Documents/110919_Resultados_ENSatJul11_U.pdf

${ }^{55}$ En la Encuesta de Satisfacción de Usuarios del Sistema de Protección Social en Salud de 2011, por ejemplo, 96.9\% de los usuarios del spss manifestaron estar "muy satisfechos" o "satisfechos" con el Seguro Popular (cNPss, "Informe de Resultados 2011", en http:/ / www.seguro-popular.salud.gob.mx/index.php?option= com_content\&view=article\&id=378\&Itemid=427, pp. 79-80). Los datos por entidad federativa también son tan favorables que resultan muy poco útiles para detectar áreas de mejora (el estado donde se reporta la proporción de satisfacción más baja en 2011 es Quintana Roo con 92.6). Los datos de estas encuestas de satisfacción contrastan de manera muy prominente con los testimonios que aparecen en la 
Por ejemplo, la Encuesta Nacional de Satisfacción de Derechohabientes Usuarios de Servicios Médicos del Imss de julio de 2011 revela que $79 \%$ de los derechohabientes que usan los servicios en todo el país reportó estar muy satisfecho o satisfecho con el servicio médico recibido.

\section{Cuadro 2}

Evolución de los desequilibrios financieros del sector salud en México, 2000-2010

\begin{tabular}{|c|c|c|c|c|}
\hline & Indicador & 2000 & 2004 & 2010 \\
\hline Nivel & Gasto en salud como porcentaje del PIв & $5.10 \%$ & $6.00 \%$ & $6.30 \%$ \\
\hline Origen & $\begin{array}{l}\text { Gasto de bolsillo como porcentaje del } \\
\text { gasto total en salud }\end{array}$ & $50.90 \%$ & $51.70 \%$ & $47.10 \%$ \\
\hline Distribución & $\begin{array}{l}\text { Relación del gasto público per cápita } \\
\text { entre aquéllos cubiertos por la } \\
\text { seguridad social y aquéllos sin } \\
\text { seguridad social }\end{array}$ & 2.1 a 1.0 & 2.1 a 1.0 & 1.2 a 1.0 \\
\hline Distribución & $\begin{array}{l}\text { Relación del gasto en salud federal per } \\
\text { cápita en el estado con el valor más alto } \\
\text { y el estado con el valor más bajo }\end{array}$ & 6.1 a 1.0 & 4.3 a 1 & 3.0 a 1.0 \\
\hline $\begin{array}{l}\text { Contribución } \\
\text { estatal }\end{array}$ & $\begin{array}{l}\text { Variabilidad en las contribuciones del } \\
\text { estado al financiamiento de la atención } \\
\text { a la salud (coeficiente de variación) }\end{array}$ & 1 & 0.8 & 0.7 \\
\hline $\begin{array}{l}\text { Asignación de } \\
\text { fondos }\end{array}$ & $\begin{array}{l}\text { Porcentaje del presupuesto de la } \\
\text { Secretaría de Salud destinado } \\
\text { inversión }\end{array}$ & $3.30 \%$ & $3.10 \%$ & $4.40 \%$ \\
\hline
\end{tabular}

Fuente: Felicia Knaul, Eduardo González-Pier, Octavio Gómez-Dantés et al., "Hacia la cobertura universal en salud: protección social para todos en México", Salud Pública de México, vol. 55, núm. 2, 2013, p. 217.

prensa con frecuencia ( $v$. gr. Emiliano Thibaut, "El terrible Seguro Popular", La Jornada, 3 de diciembre de 2011, en http:/ /www.jornada.unam.mx/2011/12/03/ politica/019a2pol) o con las declaraciones de los operadores de la política en los estados, mismos que se reportan en Mauricio Merino y Laura Flamand, Estudio para el fortalecimiento de la coordinación, administración efectiva y eficiente del Sistema de Protección Social en Salud en las entidades federativas, Reporte preparado para la Comisión Nacional de Protección Social en Salud y el Centro de Investigación y Docencia Económicas, 2011. 
En suma, el Seguro Popular ha alcanzado resultados positivos, aunque modestos, respecto a varios de sus objetivos: protección financiera (disminución en el gasto de bolsillo, sobre todo de las familias más marginadas, pero más en zonas urbanas que rurales), gasto más equitativo (entre los grupos poblacionales y entre las entidades federativas), mayores contribuciones estatales, mayor uso de los servicios sanitarios y afiliados satisfechos. Sin embargo, es importante enfatizar que estos resultados comenzaron a observarse desde el final del gobierno de Fox.

Por ejemplo, el proceso de afiliación al Seguro Popular dio prioridad a la población más pobre, pues se concentró en los grupos de ingreso más bajos (en el quintil más pobre). ${ }^{56}$ Según Gakidou y colaboradores, en 2005 el porcentaje más alto de afiliados al Seguro Popular se ubicaba en el segundo decil más bajo de ingresos familiares $(21 \%)$, mientras la proporción de afiliados disminuía en los deciles más altos. ${ }^{57}$ Ese mismo estudio muestra que la afiliación al Seguro Popular se concentró en los municipios altamente marginados (40\% de los afiliados en los deciles 2 y 3 de marginación municipal). La acertada focalización de la afiliación continuó en años posteriores, como sugiere el estudio de Felicia Knaul et al. ${ }^{58}$ de los 52.6 millones de afiliados en abril de 2012, la mayor parte se ubicaba en los cuatro deciles de menores ingresos, $35 \%$ residía en comunidades rurales y $9 \%$ pertenecía a comunidades indígenas. En síntesis, la afiliación al Seguro Popular parece haber favorecido a los grupos más pobres del país, pero ello ocurrió desde su primera etapa.

La utilización real de los servicios financiados por el Seguro Popular es otro indicador que mejoró desde el inicio de la política. A partir de la Encuesta Nacional de Ingresos y Gastos de los

${ }^{56}$ Según datos de Felicia Knaul, Héctor Arreola-Ornelas, Oscar Méndez-Carniado, Chloe Bryson-Cahn, Jeremy Barofsky, Rachel Maguire, Martha Miranda y Sergio Sesma, "Las evidencias benefician al sistema de salud: reforma para remediar el gasto catastrófico y empobrecedor en salud en México", Salud Pública de México, vol. 49, núm. 1, 2007, pp. 70-87.

${ }^{57}$ Gakidou, Lozano, González-Pier et al., art. cit.

${ }^{58}$ Knaul, González-Pier, Gómez-Dantés et al., "Hacia la cobertura universal...”. 
Hogares (ENIGH) de 2004, John Scott ${ }^{59}$ muestra que, desde los inicios del Seguro Popular, sus beneficiarios utilizaban más servicios de salud que las personas sin aseguramiento, independientemente del nivel de ingresos. Por su parte, Emmanuela Gakidou y colaboradores $^{60}$ reportan que el Seguro Popular aumentó la utilización de los servicios, especialmente entre la población con mayores necesidades sanitarias, en los estados con menores niveles de desarrollo y entre los deciles más bajos de ingreso. Según ese estudio, la probabilidad de que una persona afiliada al Seguro Popular utilizara servicios de salud era de $62 \%$, frente a $54 \%$ para una persona sin aseguramiento médico. El mismo estudio revela aumentos sustanciales, entre 2000 y 2005, en la frecuencia de diversas intervenciones ( $v . g$. hipertensión arterial, mamografías, pruebas de detección de cáncer cérvico uterino, atención por personal calificado en el parto) y un crecimiento de las tasas de egreso hospitalario.

Como ya mencionamos, es evidente que el financiamiento público en salud no ha aumentado a un ritmo consistente con la intención de la universalización, que discutiremos en la siguiente sección. De hecho, aunque el número de médicos se ha incrementado, el número de camas por habitante incluso ha descendido. El dramático crecimiento de la cobertura y del gasto de Seguro Popular se ha acompañado con incrementos mucho más paulatinos en los recursos humanos disponibles y en la infraestructura. En conjunto, esto redunda en esperas largas, servicios de baja calidad y falta de medicamentos y otros productos en las unidades médicas (que los pacientes o sus familiares, por tanto, se ven obligados a adquirir). Además, como ya mencionamos, las reducciones del gasto de bolsillo y el catastrófico son muy modestas: aproximadamente, el de bolsillo disminuye de $51 \%$ en 2000 a $47 \%$ en 2010; el catastrófico ha descendido de 3.1 a $2 \%$ del gasto total en salud. ${ }^{61}$ ¿Cuáles son los desafíos que enfrenta el Seguro Popular para ofrecer servicios de

${ }^{59}$ John Scott, "Seguro Popular: Incidence Analysis", Decentralized Service Delivery for the Poor, World Bank, en http://siteresources.worldbank.org/INTMEXICOINSPANISH/Resources/VolIIChapter3.pdf

${ }^{60}$ Gakidou, Lozano, González-Pier et al., art. cit.

61 Knaul, González-Pier, Gómez Dantés et al., "The Quest for Universal Health...". 
salud efectivos, oportunos y de calidad? ¿La solución es crear un sistema universal de salud?

\section{Los retos actuales del Seguro Popular y su Relación CON EL SISTEMA UNIVERSAL DE SALUD}

Como varios trabajos han destacado, la política social en México tiene dos componentes: primero, la provisión de beneficios asociados a los empleos formales (es decir la seguridad social) y, segundo, programas sociales que entregan beneficios a la población no asegurada. ${ }^{62}$ Sin embargo, en 2010 , aun con estos dos componentes, $39.5 \%$ del total de hogares en México y $22.3 \%$ de los más pobres no contaban con la protección de programas sociales o de la seguridad social. ${ }^{63}$ Es decir, a pesar del considerable incremento en el gasto social, que detallamos en la primera parte del artículo, y del incremento en la cobertura de los programas sociales, este sistema dual y fragmentado deja desprotegidos a casi 4 de cada 10 hogares en el país.

En este artículo, hemos destacado que la protección social en salud también se encuentra fragmentada y que es profundamente inequitativa, tanto entre las personas aseguradas y las no aseguradas, como entre los residentes de las 32 entidades del país. En un primer momento, el Seguro Popular se propuso disminuir estas inequidades, sin embargo, la discusión actual parece sugerir que la universalización de los servicios de salud en México

${ }^{62}$ Sergio Cárdenas, Édgar Ramírez, Ignacio Lozano y David Sánchez, Levantamiento del panel de la Encuesta Nacional de Afiliados al Seguro Popular. Informe final, 2012, México, CIDE, en http:/ / seguropopular.cide.edu/documents/130486/13072 6/201201_panel.pdf; Carlos Urzúa, Luis López-Calva y Eduardo Ortiz (coords.), Política social para la equidad, Ibergop-México, Porrúa, 2006.

${ }^{63}$ Se refiere a los hogares sin apoyos de Procampo y Oportunidades, aquellos donde ningún integrante cuenta con Seguro Popular y donde el jefe del hogar no cuenta con derechohabiencia a servicios médicos como prestación laboral (Consejo Nacional de Evaluación de la Política de Desarrollo Social (Coneval), Informe de Evaluación de la Política de Desarrollo Social en México 2012, México, Coneval, 2012, p. 191, en http:/ /www.coneval.gob.mx/Informes/Evaluacion/IEPDS2012/PagesIEPDSMex2012-12nov-VFinal_lowres6.pdf). 
permitiría ofrecer protección a toda la población y resolver estas inequidades. ${ }^{64}$

En este punto, conviene recapitular sobre las razones detrás de esta tendencia. México tiene el gasto en salud per cápita más bajo de todos los países miembros de la OCDE; también por abajo de Argentina y de Chile. El gasto de bolsillo, además, sigue siendo casi la mitad del gasto sanitario total (47.3\%). Incluso con el incremento del financiamiento público relacionado con la creación del Seguro Popular, el gasto de bolsillo sólo ha disminuido cuatro puntos porcentuales. ${ }^{65}$ Es claro, también, que el financiamiento del sistema sufrirá presiones adicionales por el déficit de los sistemas de pensiones de los trabajadores del sector público y el impacto general del envejecimiento de la población sobre la demanda de servicios de salud. ${ }^{66}$

En este apartado final, ofrecemos las conclusiones del artículo y discutimos brevemente las características, las ventajas y los riesgos de la universalización de los servicios de salud. En la primera parte, discutimos tres de los retos más importantes del Seguro Popular: la provisión descentralizada, la heterogeneidad de los servicios estatales de salud y la relación entre calidad de los servicios y financiamiento. En el segundo apartado, comenzamos por preguntarnos a qué tipo de sistema universal de salud apuntan las reformas del gobierno de Peña Nieto. Aunque parezca obvio, en la discusión pública no se ha definido todavía qué tipo de sistema universal se pretende construir, pues, alrededor del mundo los sistemas muestran gran variación en cuanto a carteras de servicios, rectoría, organización, participación social y financiamiento (e.g. el sistema canadiense está mucho más descentralizado que el británico, los colegios de profesionales médicos en Brasil tienen un papel más activo en la gobernanza del sistema que en Canadá, los sectores privados y social son mucho más activos en el sistema canadiense que en el británico).

\footnotetext{
${ }^{64}$ Funsalud, "Universalidad de los servicios...", op. cit.

65 Ibid., p. E21.

${ }^{66}$ Ibid., p. E26.
} 


\section{'Los retos actuales del Seguro Popular}

Incluso considerando las propuestas actuales de universalización del sistema de salud, es muy probable que la organización descentralizada del Seguro Popular se mantenga vigente. En México, la provisión estatal de los servicios públicos de salud para los no asegurados deja claro que la implementación efectiva de un sistema universal de salud requiere afinar los mecanismos de coordinación intergubernamental, con la intención de disminuir las profundas heterogeneidades entre las entidades.

De otra manera, aun con un sistema universal, las diferencias en infraestructura, recursos humanos, procesos y resultados entre los sistemas estatales de salud se traducirán en inequidades perdurables entre los servicios disponibles para los habitantes de las distintas entidades. A partir de un análisis más extenso y profundo, ${ }^{67}$ en esta sección detallamos los desafíos más importantes del Seguro Popular en los años venideros.

(i) La provisión descentralizada de los servicios de salud para los afiliados al Seguro Popular

Desde su concepción original, la implementación del Seguro Popular descansa en el principio de separar a la instancia responsable de prestar los servicios de salud a sus afiliados de la entidad encargada de financiar el sistema, con el propósito de asignar los recursos de manera más eficiente. ${ }^{68}$ En consecuencia, los regímenes estatales de protección en salud (REPSs) se concibieron como unidades administrativas responsables de cumplir dos tareas esenciales: por un lado, gestionar y administrar los recursos financieros del sistema y, por otro, coordinar la prestación de los servicios de

67 Véase Laura Flamand y Carlos Moreno-Jaimes, Seguro popular y federalismo. Un análisis de política pública, México, CIDE, 2014.

${ }^{68}$ La separación entre el financiamiento y la prestación de servicios es una recomendación generalizada entre agencias internacionales y especialistas en políticas de atención de la salud. Véase, por ejemplo, el estudio de Alexander Harding y April Preker, Innovations in Health Care Delivery: Organizational Reforms within the Public Sector, Washington, D. C., The World Bank, Health Nutrition and Population, 2000. 
atención médica en las entidades. ${ }^{69}$ Esta segunda función puede llevarse a cabo de manera directa a través de los sistemas estatales de salud o bien, de forma indirecta, a través de otros establecimientos, ya sean los sistemas estatales de otras entidades, las instituciones de la seguridad social o prestadores privados. En cualquier caso, los REPSs deben suscribir convenios de gestión con los proveedores de servicios a partir de criterios homogéneos.

Sin embargo, el desempeño de los Repss en estas funciones varía, de manera muy considerable, entre los estados. Por ejemplo, en cuanto a la gestión de los recursos financieros, durante 2010 la mayoría de los estados rebasó el tope máximo de 40\% para pago de servicios personales (establecido en los acuerdos de coordinación entre el gobierno federal y los estados), mientras que sólo diez entidades rebasaron el tope máximo de $30 \%$ para la compra de medicamentos. ${ }^{70}$ En cuanto a la coordinación de la prestación de servicios médicos, los gestores médicos son uno de los mecanismos principales para vincular a los afiliados al Seguro Popular con los proveedores de servicios, pues orientan a los pacientes y los acompañan durante su atención médica. Sin embargo, en este aspecto también se observan desigualdades importantes entre las entidades federativas: mientras que Aguascalientes y Baja California Sur tienen 3.7 y 6.2 gestores por cada 100000 afiliados, respectivamente, en Baja California y el Distrito Federal hay menos de uno. ${ }^{71}$ Finalmente, también en cuanto a la prestación de los servicios, el estudio de Gómez Robledo y asociados encuentra que la mayoría de los RePss dan prioridad a los servicios estatales de

${ }^{69}$ Esto se establece en el artículo 3 y en el capítulo II del Reglamento de la Ley General de Salud en Materia de Protección Social en Salud.

${ }^{70}$ Según el estudio de Eduardo Lazcano Ponce, Héctor Gómez Dantés, Rosalba Rojas y Francisco Garrido Latorre, Sistema de Protección Social en Salud. Evaluación externa 2012, Cuernavaca, México: Instituto Nacional de Salud Pública, 2013, el porcentaje promedio por entidad federativa destinado al rubro de servicios personales fue de $48.8 \%$ en 2013, con 22 estados que sobrepasaron el límite estatutario de $40 \%$, mientras que en el rubro de medicamentos únicamente diez estados rebasaron el tope, y sólo Guerrero y Sinaloa lo hicieron por más de 10 puntos porcentuales.

${ }^{71}$ Laura Flamand y Carlos Moreno-Jaimes, op. cit. 
salud en la compra de servicios, pese a que, como ya hemos comentado, el marco normativo los autoriza a que acudan a prestadores privados o públicos de otras entidades. ${ }^{72}$

En suma, la operación del Seguro Popular en las entidades revela diferencias significativas en los aspectos financieros, regulatorios y operativos que contravienen el principio de separación entre la provisión y el financiamiento, lo cual ocasiona problemas diversos en cuanto a la rendición de cuentas de los recursos empleados. ${ }^{73}$ Desde luego, estos problemas son consecuencia del carácter descentralizado de la implementación de esta política pública, pero, también, como hemos señalado en nuestro análisis sobre la operación del Seguro Popular a nivel estatal, ${ }^{74}$ de la situación de dependencia jerárquica de los REPSs respecto de los servicios estatales de salud. Esta situación impide, en la práctica, que los REPSS supervisen de manera efectiva los servicios de los sistemas estatales y, desde luego, facilita que los recursos se utilicen para propósitos diferentes a la atención sanitaria de los afiliados. La escasa autonomía estructural de los REPss reduce, de manera muy considerable, su influencia sobre la planeación de los sistemas, el destino de los recursos y las decisiones estratégicas.

En consecuencia, recomendamos se modifique la situación jurídica de los RePss para que se conviertan en delegaciones de la Comisión Nacional de Protección Social en Salud, con el propósito de eliminar su dependencia política y administrativa de los gobiernos estatales. Esto no significa regresar al modelo de provisión

72 Luz María González-Robledo, Gustavo Nigenda, María Cecilia GonzálezRobledo y Michael Reich, "Separación de funciones en el Sistema de Protección Social en Salud, México 2009: avances y retos”, Salud Pública de México, vol. 53, suplemento 4, 2011, pp. s416-s424.

${ }^{73}$ El trabajo de Lazcano Ponce et al., Sistema de Protección Social en Salud..., op. cit., sintetiza algunas de las observaciones que la Auditoría Superior de la Federación hizo en la mayoría de las entidades federativas en su revisión del Sistema de Protección Social en Salud, en 2010 entre las cuales destacan el pago de conceptos de nómina no autorizadas, remuneraciones a personas que no están directamente involucradas en la presentación de servicios a los afiliados, falta de transferencia de los rendimientos financieros a la cuenta bancaria del REPss, adquisición de medicamentos por encima de los precios sugeridos de referencia, entre otros.

${ }^{74}$ Laura Flamand y Carlos Moreno-Jaimes, op. cit. 
centralizada de los servicios de salud, sino evitar que en el financiamiento y en las decisiones de toda índole del Seguro Popular influyan criterios políticos o de otra índole surgidos de los gobiernos estatales. ${ }^{75}$

(ii) La heterogeneidad del marco jurídico del Seguro Popular en su operación descentralizada

En otra investigación, hemos mostrado que no existe un marco jurídico homogéneo entre las entidades federativas para garantizar la protección social en salud. ${ }^{76}$ No todas las entidades han dado a esta política pública la jerarquía legal que requiere para dar sustento jurídico suficiente a las decisiones emanadas de las instancias normativas y ejecutoras de Seguro Popular. Este hecho agrava todavía más las consecuencias negativas derivadas de la alta dependencia orgánica de los REPss, pues dejan a los operadores del Seguro Popular sin los medios jurídicos necesarios para exigir que los recursos se utilicen adecuadamente.

Las entrevistas que realizamos con funcionarios de los REPSS en 2010 y en 2013 revelan que su capacidad de gestión se incrementa, generalmente, en función de las relaciones personales que establecen con actores políticos y burocráticos, tanto estatales como municipales. ${ }^{77}$ Consideramos que es indispensable homogeneizar el marco jurídico que regula al Seguro Popular en las entidades, con el propósito de dar mayor garantía y estabilidad a su operación descentralizada.

(iii) Re-afiliación, la calidad de los servicios de salud y su relación con el financiamiento

Como ya hemos descrito, a partir de la creación del Seguro Popular, el cambio central en el financiamiento de los servicios de salud para la población no asegurada fue que las transferencias federales a las entidades responden al número de afiliados a este

${ }^{75}$ Mauricio Merino y Laura Flamand, op. cit.

${ }^{76}$ Véase Laura Flamand y Carlos Moreno-Jaimes, op. cit.

${ }^{77}$ Véanse Mauricio Merino y Laura Flamand, op. cit. y Laura Flamand y Landy Sánchez (coords. generales), Auditoría del Padrón de Afiliados del Sistema de Protección Social en Salud, México, CNPss, 2013. 
seguro público y voluntario. En una primera fase, por tanto, las entidades tuvieron como meta afiliar al mayor número de personas entre la población sin seguridad social. Sin embargo, ¿qué ocurrió, a partir de 2011, cuando se alcanzó la cobertura universal de acuerdo a los datos del gobierno federal? Dado que la póliza de afiliación tiene una duración de tres años, a partir de 2012 y hasta la fecha, los recursos se asignan a las entidades de acuerdo al número de afiliados vigentes; es decir, ahora la meta de los REPss es reafiliar al mayor número de personas y, desde luego, promover afiliaciones nuevas.

La reafiliación es un proceso muy complicado y costoso para las entidades federativas, pues los afiliados no suelen acudir a renovar sus pólizas a menos de que requieran atención médica y, con frecuencia, reportan atención de baja calidad o desabasto de medicamentos como razones para no reafiliarse. Los REPss, en consecuencia, se han visto obligados a implementar estrategias de promoción y reafiliación muy proactivas; las más dominantes son brigadas que visitan a los afiliados en sus domicilios. Estas visitas son muy complejas, sobre todo, en zonas urbanas donde no es sencillo localizar a los afiliados en días hábiles. ${ }^{78}$

Lo más grave, sin embargo, es que el financiamiento de Seguro Popular no se encuentra asociado a la efectividad, la oportunidad o la calidad de los servicios de salud. Es decir los sistemas estatales de salud siguen recibiendo los recursos financieros para atender a la población afiliada sin importar si previenen o curan sus padecimientos.

Incluso con el muy considerable incremento de la cobertura de servicios de salud asociado con la creación de Seguro Popular, es necesario enfatizar que esta afiliación es nominal y no necesariamente se relaciona con el acceso real a los servicios de salud, con que se usen de manera efectiva o, incluso, con que la población conozca de su afiliación o de sus derechos. ${ }^{79}$

De hecho, la información sobre la calidad de los servicios de salud provistos mediante el Seguro Popular es relativamente

${ }^{78}$ Laura Flamand y Landy Sánchez, Auditoría del Padrón de Afiliados..., op. cit.

${ }^{79}$ Loc. cit. y Funsalud, "Universalidad de los servicios...", op. cit., p. E20. 
escueta. ${ }^{80}$ Es necesario conocer con más detalle en qué medida los sistemas estatales ofrecen servicios accesibles, confiables y oportunos a la población afiliada. Los siete estudios de caso que presentamos en otro trabajo ${ }^{81}$ demuestran que los sistemas estatales no comparten los mismos atributos requeridos para garantizar una provisión de calidad en sus servicios de salud. Los datos que hemos presentado hablan por sí mismos: la eficacia de las instancias operativas en su integración de los expedientes de la población afiliada ha sido baja en general y con diferencias extraordinariamente amplias entre los estados. Hay también variaciones significativas en cuanto al grado de conocimiento de las intervenciones del CAUSES entre los operadores de los servicios de salud. Y, en general, la mayor parte de los factores relacionados con los procesos de información, planeación, supervisión y evaluación del Seguro Popular se caracterizan por la heterogeneidad con la cual operan en las entidades federativas.

El problema central es que las decisiones de financiamiento de Seguro Popular no se vinculan con los estándares de calidad bajo los cuales operan los servicios de salud en los estados. Sabemos bien que uno de los objetivos fundamentales de la política es, desde su inicio, que la transferencia de recursos financieros responda de manera dominante al número de afiliados de manera que el financiamiento atienda a la demanda y no a la capacidad instalada. Sin embargo, nuestros hallazgos sugieren que los usuarios no tienen garantía sobre la calidad de los servicios que reciben. Desde luego, para condicionar la entrega de recursos financieros al cumplimiento de estándares de efectividad y calidad en los servicios es crucial fortalecer los sistemas de planeación, evaluación, información y supervisión del Seguro Popular, procurando reducir la gran heterogeneidad que hoy existe entre las entidades. Nuestra recomendación de que los RePss salgan de la esfera administrativa de los gobiernos estatales para convertirse en órganos desconcentrados

80 Véase, por ejemplo, Hortensia Reyes-Morales, Sergio Flores-Hernández, Alma Sauceda-Valenzuela y otros, "Percepción de los usuarios sobre la calidad de la atención ambulatoria en servicios de salud en México", Salud Pública de México, vol. 55, supl. 2, 2013, pp. s100-s105.

${ }^{81}$ Laura Flamand y Carlos Moreno-Jaimes, op. cit. 
de la Comisión Nacional sería una decisión consistente con este objetivo, pero tendría que complementarse con una revisión de las condiciones bajo las cuales se suscriben los anexos a los convenios del Sistema para que el financiamiento responda de manera clara a criterios de calidad en los procesos y en la entrega de los servicios. ${ }^{82}$

\section{¿Un sistema universal de salud en México?}

El tema de la universalización de los servicios de salud ha vuelto a cobrar vigencia en la discusión política y académica en nuestro país. ${ }^{83}$ La propuesta de establecer un sistema único de salud (que implica eliminar el sistema dual que hoy existe) suele descansar en dos argumentos principales. El primero subraya que, aun con los indudables logros del Seguro Popular como mecanismo de protección financiera para las personas sin acceso a la seguridad social (especialmente para la población de ingresos más bajos), el problema de desigualdad sigue siendo el elemento más distintivo del sistema de salud mexicano. Aun cuando la regresividad del gasto en salud de México ha disminuido considerablemente a raíz de la creación del Seguro Popular, nuestro país sigue estando dentro de los países de la región con mayor desigualdad en su sistema de atención sanitaria. ${ }^{84}$ Según este diagnóstico, la inequidad que caracteriza al sistema de salud mexicano persistirá, a menos que se lleve a

${ }^{82}$ Mauricio Merino y Laura Flamand, op. cit.

${ }^{83}$ Probablemente, el trabajo que renovó el interés en el tema -y sobre el cual ha girado mucha de la discusión reciente- fue el de Santiago Levy, Good Intentions, Bad Outcomes: Social Policy, Informality, and Economic Growth in Mexico, Washington, D. C., Brookings Institution Press, 2008. Otras propuestas similares, algunas de las cuales difieren en ciertos aspectos de diseño, pueden consultarse Centro de Estudios Espinosa Yglesias, El México del 2012: Reformas a la hacienda pública y al sistema de protección social, México, Centro de Estudios Espinosa Yglesias, 2012; Programa de las Naciones Unidas para el Desarrollo (PNUD), Informe sobre Desarrollo Humano en México 2011, en http://www.undp.org.mx/IMG/pdf/Informe_sobre_Desarrollo_Humano_Mexico_2011.pdf; también: Grupo Nuevo Curso de Desarrollo, Lineamientos de política para el crecimiento sustentable y la protección social universal, 2012, en http:/ /www.nuevocursodedesarrollo.unam.mx/MFC2012.pdf

${ }^{84}$ PNUD, op. cit. 
cabo una reforma profunda que desaparezca la dualidad existente entre las instituciones de seguridad social que atienden a los trabajadores del sector formal y las políticas de protección social que cubren a los trabajadores informales, entre ellas el Seguro Popular.

Santiago Levy ha desarrollado un segundo argumento a favor de la creación de un sistema universal, no sólo de atención a la salud, sino de protección social en general. Levy sostiene que la política social de México, erigida a partir del tipo de inserción laboral de las personas (formal o informal), ha dado lugar a un sistema dual que no ha logrado combatir la pobreza, redistribuir efectivamente el ingreso, ni mucho menos fomentar la productividad y el crecimiento económico. ${ }^{85}$

Según Levy, de los casi 43 millones de trabajadores existentes en México, ${ }^{86}$ únicamente 17 millones pertenecen al sector formal de la economía, lo cual implica que sólo una minoría tiene acceso a los beneficios completos del régimen de la seguridad social: los seguros médico, de vida y contra riesgos de trabajo, pensiones por retiro, así como guarderías, créditos de vivienda y servicios culturales y deportivos. El resto de los trabajadores pertenecen al sector informal, es decir, se trata de trabajadores por cuenta propia o comisionistas, pero también de trabajadores contratados ilegalmente por empresas que no los inscriben a la seguridad social. El conjunto mayoritario, el de los trabajadores informales, no cuenta con la protección de la seguridad social, pero tiene acceso a diversos programas sociales (incluido el Seguro Popular), financiados sobre todo con recursos del erario público, que pueden adquirirse voluntariamente, por separado y de manera gratuita. Levy sostiene que estas políticas sociales "no contributivas" (es decir no financiadas con cuotas obrero-patronales) han fomentado que tanto las empresas como los trabajadores opten por la informalidad, pues los trabajadores tienen la alternativa de recibir la cobertura de los programas de protección social gratuita sin tener que pagar el costo de la seguridad social (la cual es de aproximadamente $25 \%$ del salario).

${ }^{85}$ Levy, op. cit.

${ }^{86}$ Las cifras estimadas por Santiago Levy, op. cit., corresponden a 2006. 
El resultado de este esquema dual de protección social es que genera poca redistribución del ingreso, baja productividad económica y un crecimiento muy limitado. ${ }^{87}$ De ahí, Levy deriva la recomendación de universalizar todo el sistema de protección social con financiamiento de impuestos generales (en específico con el Impuesto al Valor Agregado a todos los bienes y servicios) de manera que no sean las empresas quienes asuman el costo de la protección social, sino la sociedad en su conjunto. ${ }^{88}$ La recaudación de este IVA generalizado se etiquetaría totalmente para ofrecer a todos los mexicanos un esquema de protección social consistente en una pensión de retiro, y seguros de salud, de vida, de invalidez, de riesgos de trabajo y de desempleo.

Además del estudio de Levy, durante la elaboración de este artículo revisamos tres propuestas de universalización de los servicios de salud en México: reforma hacendaria y social del Centro de Estudios Espinoza Yglesias, reforma legislativa asociada a la universalización de los servicios de salud del Centro de Investigación y Docencia Económicas y el estudio sobre la universalidad de los servicios de salud en México de la Fundación Mexicana de la Salud (Funsalud). En los apartados siguientes analizamos brevemente la

${ }^{87}$ Es importante aclarar que la hipótesis defendida por Levy de que los programas de protección social fomentan la informalidad ha sido cuestionada por otros investigadores. Autores como Oliver Azuara e Ioana Marinescu ("Informality and the expansion of social protection programs", Documento de trabajo del Munich Personal RePEc Archive (MPRA), 2011, en http://mpra.ub.uni-muenchen. de/ 35073/) y Rodrigo Barros ("Wealthier But Not Much Healthier: Effects of a Health Insurance Program for the Poor in Mexico", Documento de discussion del Stanford Institute for Economic Policy Research, 2008, en http:/ / economics.stanford. edu/files/JMP_RBarros.pdf) no encuentran evidencia de que el Seguro Popular haya incentivado la informalidad en el mercado laboral. Por su parte, el trabajo de Gerardo Esquivel y Juan Luis Ordaz, “¿Es la política social una causa de la informalidad en México?”, Ensayos Revista de Economía, vol. 27, núm. 1, 2008, pp.132 , demuestra que la política social no es la causa primordial de la informalidad en la economía.

${ }^{88} \mathrm{Si}$ bien Santiago Levy, op. cit., defiende la opción de financiar al sistema universal de protección social a través del IVA generalizado, hay quienes consideran que sería mejor hacerlo mediante el impuesto sobre la renta (ISR), a fin de reducir sus posibles impactos regresivos sobre la distribución del ingreso (Grupo Nuevo Curso de Desarrollo, op. cit.). 
propuesta de Funsalud ${ }^{89}$ por dos razones: primera, es la única propuesta que se concentra en los servicios de salud exclusivamente y, en consecuencia, presenta el análisis más detallado; y, segunda, Mercedes Juan, la actual secretaria de Salud del gobierno federal, dirigió el grupo que elaboró la propuesta de Funsalud, por tanto, muy probablemente, esta propuesta animará la reforma de Peña Nieto.

La propuesta de Funsalud reconoce que el objetivo básico de la reforma es eliminar las desigualdades en la cobertura de los servicios de salud. De manera abierta se afirma que "la fragmentación del sistema no ha permitido el ejercicio universal e igualitario de ese derecho". ${ }^{90}$ Para la universalización, el reto más complejo, por tanto, consiste en unificar los tres seguros públicos principales, es decir, el Seguro Popular (38.5\% del total de la población), el IMSS $(32.2 \%)$ y el IssSTE $(6 \%) .{ }^{91}$

El corazón de la reforma de Funsalud consiste en separar las funciones del sistema sanitario. Es decir, la Secretaría de Salud se ocuparía de la rectoría y del financiamiento a partir de impuestos generales, ${ }^{92}$ mientras que la prestación de servicios se realizaría con redes de atención de instituciones públicas y privadas (IMss, IsssTe y los servicios estatales de salud). Además, en la propuesta se enfatiza que los ciudadanos podrán elegir, de manera libre, entre los proveedores de servicios.

El estudio de Funsalud es un diagnóstico muy cuidadoso, pero sólo un primer esbozo de los cambios legislativos, organizacionales y culturales asociados a la reforma. Los aspectos que es necesario desarrollar y resolver son innumerables. Por ahora ofrecemos sólo un trío: a) ¿Cómo se definirá el paquete universal de servicios

${ }^{89}$ Funsalud, "Universalidad de los servicios de salud en México", Salud Pública de México, vol. 55, 2013, pp. EE1-EE64.

90 Ibid., p. E4.

${ }^{91}$ Los datos son cálculos de Juan Pablo Gutiérrez y Mauricio Hernández-Ávila, "Cobertura de protección en salud...", op. cit., a partir de los resultados de la Ensanut 2012. De acuerdo a estos datos, en 2012, 21.4\% de la población total en México no contaba con protección social en salud.

${ }^{92}$ Lo cual significa, en la práctica, abandonar, por lo menos parcialmente, los regímenes contributivos de la seguridad social. 
de salud y cómo se resolverán los casos donde la cobertura de la seguridad social supere a la del paquete universal? b) Dado que el sistema supone que los ciudadanos podrán elegir entre los proveedores, ¿cómo se resolverá el exceso de demanda que puede esperarse en las unidades médicas que ofrecen servicios con mayor efectividad y calidad? c) Considerando que, en principio, el costo de las intervenciones será homogéneo, ¿cómo se determinarán los sueldos y las prestaciones de los trabajadores de la salud de las diversas instituciones proveedoras, a saber, del IMSS, del IssSTE y de los SESA? ${ }^{93}$

Otro asunto que requiere abordarse con más detalle es el papel del sector privado en un sistema universal de salud. Nuestra revisión del Sistema Único de Salud (sus) de Brasil reveló con toda claridad que los problemas de desigualdad que todavía lo caracterizan están íntimamente relacionados con la alta dependencia que el sus tiene respecto al sector privado. ${ }^{94}$ La evidencia indica que los proveedores privados (especialmente en el segundo nivel de atención) suelen aplicar estándares duales de atención, favoreciendo a los usuarios con planes privados de aseguramiento médico, problema que se exacerba tomando en cuenta que el financiamiento público del sus tiene un papel limitado (39\% del gasto total en salud). Por tanto, en México es necesario discutir, primero, si los prestadores de servicios privados de salud podrían acceder competitivamente a los recursos públicos que financiarían al sistema de protección social universal; segundo, si el aseguramiento médico privado sería un sustituto o un complemento al sistema público de salud y, tercero, qué mecanismos regulatorios tendrían que adoptarse para evitar el fenómeno de segregación de usuarios que se ha dado en casos como el brasileño.

Lo que más interesa en este artículo, sin embargo, es resaltar que la propuesta de universalización de Funsalud, igual que la de Seguro Popular en sus orígenes, presta escasa atención a los servicios estatales de salud y a su patente heterogeneidad. Muy

${ }^{93}$ Esta última pregunta, desde luego, refiere a cómo se lidiará con los poderosos sindicatos de la Secretaría de Salud, el imss y el Issste.

${ }^{94}$ Laura Flamand y Carlos Moreno-Jaimes, op. cit., cap. I. 
probablemente, el esquema de descentralización heredado de la década de 1990, sobre el cual se ha montado, por mandato jurídico, el Seguro Popular, continuará siendo la base organizativa de la provisión de los servicios de salud, aunque, como ya hemos mencionado, es necesario modificar los mecanismos de implementación para mejorar la calidad de los servicios.

En breve, incluso si un sistema universal de salud elimina las desigualdades asociadas con la fragmentación entre los servicios para las personas con y sin seguridad social, la protección social en salud seguirá siendo profundamente inequitativa entre las entidades federativas si no hay un esfuerzo explícito y claro para fortalecer los servicios estatales de salud estableciendo los incentivos necesarios para que presten servicios efectivos, oportunos y de calidad.

\section{BibLIOGRAFÍA}

Aguilera, Nelly y Mariana Barraza-Llorenz, "FASSA: análisis sobre equidad y alternativas de asignación”, en Carlos Chiapa y César Velázquez (eds.), Estudios del Ramo 33, México, Coneval-Colmex, 2011.

Auditoría Superior de la Federación, "Comisión Nacional de Protección Social en Salud. Seguro Popular. Auditoría de Desempeño”, en http:// www.asf.gob.mx/Trans/Informes/IR2011i/Grupos/Desarrollo_ Social/2011_0426_a.pdf

Ávila-Burgos, Leticia, Edson Serván-Mori, Verónica Wirtz, Sandra SosaRubi y Aaron Salinas-Rodríguez, "Efectos del Seguro Popular sobre el gasto en salud en hogares mexicanos a diez años de su implementación”, Salud Pública de México, vol. 55, suplemento 2, 2013, pp. s91-s99.

Azuara, Oliver e Ioana Marinescu, "Informality and the Expansion of Social Protection Programs", Documento de trabajo del Munich Personal RePEc Archive (MPRA), 2011, en http://mpra.ub.uni-muenchen.de/35073/

Banco Mundial, World Development Indicators, en http://data.worldbank. $\mathrm{org} /$ data-catalog/world-development-indicators, 2014.

Barros, Rodrigo, "Wealthier But Not Much Healthier: Effects of a Health Insurance Program for the Poor in Mexico", Documento de discussion del Stanford Institute for Economic Policy Research, 2008, en http://economics.stanford.edu/files/JMP_RBarros.pdf 
Bizberg, Ilán y Laura Flamand, "Grandes tendencias y pequeños avances en las políticas sociales (2000-2012)”, en Ana Covarrubias (ed.), Doce años de gobierno del PAN, México, El Colegio de México, en dictamen.

Cárdenas, Sergio, Édgar Ramírez, Ignacio Lozano y David Sánchez, Levantamiento del panel de la Encuesta Nacional de Afiliados al Seguro Popular. Informe final, 2012, México, Centro de Investigación y Docencia Económicas, en http://seguropopular.cide.edu/documents/13048 6/130726/201201_panel.pdf

Centro de Estudios de Finanzas Públicas (CEFP), Evolución del Gasto Social 2003-2010, México, CEFP, 2011, en http://www.cefp.gob.mx/intr/ edocumentos/pdf/cefp/2009/cefp0782009.pdf

Centro de Estudios Espinosa Yglesias, El México del 2012: Reformas a la hacienda pública y al sistema de protección social, México, Centro de Estudios Espinosa Yglesias, 2012.

Centro de Estudios Sociales y de Opinión Pública, Carpeta informativa. Iniciativa de reforma a la ley del ISSSTE, 2007, en http:/ / archivos.diputados.gob.mx/Centros_Estudio/Cesop/ISSSTE_2.pdf

Comisión Económica para América Latina y el Caribe (CEPAL), Statistical Yearbook for Latin America and the Caribbean, Santiago, CEPAL, 2011, en http:/ / www.eclac.cl/publicaciones/xml/7/45607/1.pdf

—, Panorama social de América Latina, CEPAL, 2012, en http:/ / www. eclac.org/publicaciones/xml/5/48455/PanoramaSocial2012DocIRev.pdf

- Gasto Social en América Latina y el Caribe, Portal de inversión social en la región, CEPAL, 2014, en http://dds.cepal.org/gasto/indicadores

Comisión Nacional de Protección Social en Salud (CNPSS), Informe de resultados 2009, en http://www.seguro-popular.salud.gob.mx/index. php?option $=$ com_content\&view $=$ article\&id $=378 \&$ Itemid $=427$

— Informe de transferencias a las entidades federativas 2010, en http:/ / www.seguro-popular.salud.gob.mx

—, "Informe de Resultados 2011", en http:/ /www.seguro-popular. salud.gob.mx/index.php?option=com_content\&view=article\&id=37 $8 \&$ Itemid $=427$

salud.gob.mx/index.php?option=com_content\&view=article\&.id=37 8\&Itemid $=427$

, "Informe de Resultados enero-junio 2013", en http://www.se- 
guro-popular.salud.gob.mx/index.php?option=com_content\&view= article\&id $=378 \&$ Itemid $=427$

Consejo Nacional de Evaluación de la Política de Desarrollo Social (Coneval), Anexo Estadístico. Medición de la pobreza, Estados Unidos Mexicanos, 2010, en http://web.coneval.gob.mx/Paginas/principal.aspx.

-, Informe de Evaluación de la Política de Desarrollo Social en México 2012, México, Coneval, 2012, en http://www.coneval.gob.mx/ Informes/Evaluacion/IEPDS2012/Pages-IEPDSMex2012-12nov-VFinal_ lowres6.pdf

Esquivel, Gerardo, y Juan Luis Ordaz, “¿Es la política social una causa de la informalidad en México?”, Ensayos Revista de Economía, vol. 27, núm. 1, 2008, pp.1-32.

Flamand, Laura, "Las perspectivas del nuevo federalismo: el sector salud. Las experiencias en Aguascalientes, Guanajuato y San Luis Potosí”, CIDE-DTAP, 55, México, 1997.

Flamand, Laura y Carlos Moreno-Jaimes, Seguro popular y federalismo. Un análisis de política pública, México, Centro de Investigación y Docencia Económicas, 2014.

Flamand, Laura y Landy Sánchez (coords. generales), Auditoría del Padrón de Afiliados del Sistema de Protección Social en Salud, México, cNPSs, 2013.

Fundación Mexicana para la Salud (Funsalud), "Universalidad de los servicios de salud en México”, Salud Pública de México, vol. 55, 2013, pp. EE1-EE64.

Gakidou, Emmanuela, Rafael Lozano, Eduardo González-Pier, Jesse Abbott-Klafter, Jeremy Barofsky, Chloe Bryson-Cahn, Deniss Feehan, Diana Lee, Héctor Hernández-Llamas y Christopher Murray, "Evaluación del impacto de la reforma mexicana de salud 2001-2006: un informe inicial”, Salud Pública de México, vol. 49, sup. 1, 2007, pp. 88-109. Galárraga, Omar, Sandra Sosa-Rubi, Aarón Salinas-Rodriguez y Sergio Sesma-Vazquez, "Health Insurance for the poor: impact on catastrophic and out-of-pocket health expenditures in Mexico", European Journal of Health Economics, vol. 11, núm. 5, 2010, pp. 437-447.

Gómez Dantés, Octavio, Sergio Sesma, Víctor Becerril et al., "Sistema de Salud en México”, Salud Pública de México, 2011, vol. 53, núm. 2, pp. 220-233.

González Pier, Eduardo, Mariana Barraza, Cristina Gutiérrez y Armando 
Vargas, (coords.), Sistema de Protección Social en Salud: Elementos conceptuales, financieros y operativos, México, Secretaría de Salud, FcE, 2006.

González-Robledo, Luz María, Gustavo Nigenda, María Cecilia González-

Robledo y Michael Reich, "Separación de funciones en el Sistema de Protección Social en Salud, México 2009: avances y retos”, Salud Pública de México, vol. 53, suplemento 4, 2011, pp. s416-s424.

Grogger, Jeoffrey, Tamara Arnold, Ana Sofía León y Alejandro Ome, "Efectos del Seguro Popular sobre el gasto en salud, utilización de servicios médicos y nivel de salud, y resultados de largo plazo del experimento del Seguro Popular en México y evidencia de la Encuesta Nacional de Ingresos y Gastos de los Hogares (ENIGH)”, México, Centro de Investigación y Docencia Económicas, 2011.

Grogger, Jeoffrey, Tamara Arnold, Ana Sofía León y Alejandro Ome, "Heterogeneity in the Effect of Public Health Insurance on Catastrophic Out-of-Pocket Health Expenditures: The Case of Mexico", Health Policy and Planning, junio de 2014, pp. 1-7.

Grupo Nuevo Curso de Desarrollo, Lineamientos de política para el crecimiento sustentable y la protección social universal, 2012, en http:/ / www.nuevocursodedesarrollo.unam.mx/MFC2012.pdf

Gutiérrez, Juan Pablo y Mauricio Hernández-Ávila, “Cobertura de protección en salud y perfil de la población sin protección en México, 20002012”, Salud Pública de México, vol. 55, supl. 2, 2013, pp. s83-s90.

Harding, Alexander y April Preker, Innovations in Health Care Delivery: Organizational Reforms within the Public Sector, Washington, D. C., The World Bank, Health Nutrition and Population, 2000.

Instituto Mexicano del Seguro Social (IMss), Encuesta Nacional de Satisfacción de Derechohabientes Usuarios de Servicios Médicos del IMSS, 2011, en http://www.imss.gob.mx/servicios/encuesta/Documents/110919_ Resultados_ENSatJul11_U.pdf

Instituto Nacional de Estadística y Geografía (INEGI), Principales resultados del Censo de Población y Vivienda 2010, en http://www.inegi.org.mx/ default.aspx

Knaul, Felicia, Eduardo González-Pier, Octavio Gómez Dantés, David García-Junco, Héctor Arreola-Ornelas, Mariana Barraza-Lloréns, Rosa Sandoval, Francisco Caballero, Mauricio Hernández-Avila, Mercedes Juan, David Kershenobich, Gustavo Nigenda, Enrique Ruelas, Jaime Sepúlveda, Roberto Tapia, Guillermo Soberón, Salomón Chertorivski, 
Julio Frenk, "The Quest for Universal Health Coverage: Achieving Social Protection for All in Mexico", The Lancet, vol. 380, num. 9849, 2012, en http://www.thelancet.com/journals/lancet/issue/vol380 no9849/PIIS0140-6736(12)X6041-

, "Hacia la cobertura universal en salud: protección social para todos en México", Salud Pública de México, vol. 55, núm. 2, 2013, pp. 207-235.

Knaul, Felicia, Héctor Arreola-Ornelas, Oscar Méndez-Carniado, Chloe Bryson-Cahn, Jeremy Barofsky, Rachel Maguire, Martha Miranda y Sergio Sesma, Las evidencias benefician al sistema de salud: reforma para remediar el gasto catastrófico y empobrecedor en salud en México", Salud Pública de México, vol. 49, num. 1, 2007, pp. 70-87.

Knaul, Felicia, y Julio Frenk, "Health Insurance in Mexico: Achieving Universal Coverage through Structural Reform", Health Affairs, 2005, vol. 24, núm. 6, pp. 1467-1476.

Lazcano Ponce, Eduardo, Héctor Gómez Dantés, Rosalba Rojas y Francisco Garrido Latorre, Sistema de Protección Social en Salud. Evaluación externa 2012, Cuernavaca, México, Instituto Nacional de Salud Pública, 2013.

Levy, Santiago, Good Intentions, Bad Outcomes: Social Policy, Informality, and Economic Growth in Mexico, Washington, D. C., Brookings Institution Press, 2008.

Merino, Mauricio y Laura Flamand, Estudio para el fortalecimiento de la coordinación, administración efectiva y eficiente del Sistema de Protección Social en Salud en las entidades federativas, Reporte preparado para la Comisión Nacional de Protección Social en Salud y el Centro de Investigación y Docencia Económicas, 2011.

Moreno Jaimes, Carlos, "La descentralización del gasto en salud en México: una revisión de sus criterios de asignación”, México, CIDE, 2000. (Documento de Trabajo de la DAP, núm. 95)

Organización Mundial de la Salud (oms), 2010, Informe sobre la salud en el mundo: la financiación de los sistemas de salud: el camino hacia la cobertura universal, Ginebra, oms en http://www.who.int/whr/2010/es/ index.html

Organization for Economic Co-operation and Development (OECD), Health Data 2013, en http://www.oecd.org/els/health-systems/oecdhealthdata2013-frequentlyrequesteddata.htm 
Ortiz, Mauricio, El Seguro Popular. Una crónica de la democracia mexicana, México, Secretaría de Salud-Funsalud-INSP-FCE, 2006.

Presidencia de la República, Sexto Informe de Gobierno, 2012. Anexo Estadístico, en http://calderon.presidencia.gob.mx/

Programa de las Naciones Unidas para el Desarrollo (PNUD), Informe sobre Desarrollo Humano en México 2011, en http:/ / www.undp.org.mx/IMG/ pdf/Informe_sobre_Desarrollo_Humano_Mexico_2011.pdf

Reyes-Morales, Hortensia, Sergio Flores-Hernández, Alma Sauceda-Valenzuela y otros, "Percepción de los usuarios sobre la calidad de la atención ambulatoria en servicios de salud en México", Salud Pública de México, vol. 55, supl. 2, 2013, pp. s100-s105.

Secretaría de Salud, Cirugía extramuros: ¿Quiénes somos?, en http://www. salud.gob.mx/unidades/dgpfs/micsitio/cirextra/quienes/htmprese.htm

Scott, John, "Seguro Popular: Incidence Analysis", Decentralized Service Delivery for the Poor, World Bank, en http://siteresources.worldbank. org/INTMEXICOINSPANISH/Resources/VolIIChapter3.pdf

Sistema Integral de Información de Padrones de Programas Gubernamentales (SIIPP-G), Unidad de Evaluación de la Gestión y el Desempeño Gubernamental, Secretaría de la Función Pública, Resumen de hallazgos y resultados primer semestre 2013, en http://www.funcionpublica.gob. $\mathrm{mx} /$ web/doctos/ua/ssfp/uegdg/siippg/resumen_siippg_1er_se mestre_2013_ace.pdf

Sistema Nacional de Información en Salud (sinais), Boletín de Información Estadística, en http://www.sinais.salud.gob.mx/publicaciones/ bie. html

, Poblaciones 1990-2012 (total y condición de aseguramiento), en http://www.sinais.salud.gob.mx/demograficos/index.html

— Indicadores de recursos financieros, 1990-2009, en http://www.sinais.salud.gob.mx/recursosfinancieros/index.html

—_, Gasto público, 1990-2009 en http://www.sinais.salud.gob.mx/ recursosfinancieros/index.html

Soberón, Guillermo et al., "Health Services Decentralization in Mexico: Formulation, Implementation and Results of Policy", Health Policy and Planning, vol. 4, 1989, pp. 301-313.

Sosa-Rubi, Sandra, Aarón Salinas-Rodriguez y Omar Galárraga, "Impacto del Seguro Popular en el gasto catastrófico y de bolsillo en el México 
rural y urbano, 2005-2008”, Salud Pública de México, vol. 53, 2011, pp. $425-435$.

Sosa-Rubí, Sandra, Omar Galárraga y Jeffrey Harris, "Heterogeneous Impact of the 'Seguro Popular' Program on the Utilization of Obstetrical Services in Mexico, 2001-2006: A Multinomial Probit Model with a Discrete Endogenous Variable”, Journal of Health Economics, vol. 28, núm. 1, 2009, pp. 20-34.

Thibaut, Emiliano, "El terrible Seguro Popular", La Jornada, 3 de diciembre de 2011, en http:/ / www.jornada.unam.mx/2011/12/03/politica /019a2pol

Urzúa, Carlos, Luis López-Calva y Eduardo Ortiz (coords.), Politica social para la equidad, Ibergop-México, Porrúa, 2006.

World Health Organization (wHO), Global Health Observatory Data Repository, en http://apps.who.int/ghodata/

Yunes, Miguel Ángel, La reforma del ISSSTE: un cambio necesario, México, FCE, 2009. 